\title{
Análisis psicométrico del Cuestionario de Honey y Alonso de Estilos de Aprendizaje (CHAEA) con los modelos de la Teoría Clásica de los Tests y de Rasch
}

Luis Miguel Escurra Mayaute Universidad de Lima

Recibido: 19 de mayo del 2011 / Aprobado: 15 de julio del 2011

Se analiza el cuestionario de Honey y Alonso de estilos de aprendizaje (CHAEA) con los modelos psicométricos de la Teoría Clásica de los Test (TCT) y el Modelo de Rasch de la Teoría de la Respuesta al Ítem en estudiantes universitarios de Lima metropolitana. Para obtener la muestra de 830 estudiantes, se utilizó un muestreo estratificado de acuerdo con el tamaño de las universidades seleccionadas, el de las facultades y el año de estudio. Los resultados indicaron que el CHAEA funciona adecuadamente bajo los supuestos de la TCT, pero no cumple con los requerimientos teóricos del Modelo de Rasch.

estilos de aprendizaje / teoría clásica de los test/ teoría de la respuesta al ítem / modelo de Rasch

Analysis of the Questionnaire of Learning Styles of Honey-Alonso (CHAEA) with the Classical Test Theory psychometric model and the Rasch Model of the Item Response Theory

This study analyzes the Questionnaire of Learning Styles of Honey-Alonso (CHAEA) utilizing the Classical Test Theory psychometric model and the Rasch Model of the Item Response Theory in university students in metropolitan Lima. The sample consisted in 830 university students recruited using the stratified sample according to university size, school size and year of study. The HoneyAlonso Questionnaire was utilized. The statistical analysis was realized in three stages: psychometric analysis to determine validity and reliability, psychometric analysis with the Rasch model, and comparative analysis. Results indicate that the CHAEA functions adequately based on the assumptions made by the Classical Test Theory, but do not fulfill the theoretical requirements demanded by the Rasch model.

learning styles / classical test theory / item response theory / Rasch model

Correo electrónico: lescurra@correo.ulima.edu.pe 


\section{INTRODUCCIÓN}

En el desarrollo de la Psicología como ciencia, la medición a través de pruebas ha jugado un papel relevante, pues no solo ha permitido evaluar y diagnosticar tanto de manera individual como grupal, sino que además ha facilitado la toma de decisiones profesionales, especialmente en el campo de la Psicología Educativa.

Uno de los procedimientos utilizados para mejorar la calidad de la enseñanza universitaria ha sido el optimizar el aprendizaje autorregulado, especialmente a través del desarrollo de los estilos de aprendizaje (Zabalza, 2002).

Los objetivos planteados en la enseñanza en el nivel superior suponen la importancia de la autorregulación en los estudiantes universitarios como una de las condiciones para un aprendizaje de calidad; así lo resaltan las diferentes teorías cognitivas del aprendizaje.

El éxito y el fracaso académico en el ámbito universitario es un tema que adquiere progresiva importancia en la investigación educativa moderna y sobre el que hay acuerdo en cuanto a ubicar su origen en la confluencia de múltiples factores: psicológicos, sociales y económicos, entre los que destacan la existencia de diversos estilos de aprendizaje y el uso de inadecuadas estrategias, lo que se ve reflejado en un determinado rendimiento académico.
En este contexto el Cuestionario de Honey y Alonso de estilos de aprendizaje (CHAEA) ha sido utilizado por diversos autores como un instrumento de medida aplicado para identificar los diferentes tipos de estilos de aprendizaje que presentan los estudiantes (Alonso, Gallego \& Honey, 2002; Beltrán, 1996; Honey \& Mumford, 1986), lo cual amerita la revisión de sus propiedades psicométricas tanto bajo el modelo clásico como de los modelos contemporáneos.

\section{El modelo de estilos de aprendizaje de Honey y Alonso}

Una de las principales definiciones de los estilos de aprendizaje corresponde a la desarrollada por Alonso, Gallego y Honey (2002, p. 48), quienes indican:

Desde nuestro punto de vista, una de las definiciones más claras y ajustadas es la que propone Keefe (1988) y que hacemos nuestra, los estilos de aprendizaje son los rasgos cognitivos, afectivos y fisiológicos, que sirven como indicadores relativamente estables, de cómo los discentes perciben, interaccionan y responden a sus ambientes de aprendizaje.

El aprendizaje, entendido como construcción de la experiencia, es un proceso cíclico, de acuerdo con Kolb (en Witkin y Goodenoug, 1991) un aprendizaje eficaz necesita cuatro etapas: experiencia concreta, observación reflexiva, conceptualización abstracta 
y experimentación activa. Esto supone experimentar, reflexionar, elaborar hipótesis y verificarlas.

El modelo de estilos de aprendizaje elaborado por Kolb (1984) supone que para aprender algo se debe trabajar o procesar la información que se recibe mediante: a) una experiencia directa y concreta, y b) una experiencia abstracta, que es la que se tiene cuando se lee información acerca de algo o cuando la información es contada.

Las experiencias que se tienen, concretas o abstractas, se transforman en conocimiento cuando se elaboran de alguna de estas dos formas: a) reflexionando y pensando sobre ellas, y b) experimentando de manera activa con la información recibida.

En la práctica, las personas tienden a desarrollar una o dos de dichas cuatro fases, por ello se pueden establecer cuatro tipos de alumnos, dependiendo de la fase en la que prefieran trabajar:

a) Divergentes.- $\mathrm{Si}$ se basan en las experiencias concretas y la observación reflexiva.

b) Convergentes.- Si utilizan la conceptualización abstracta y la experimentación activa.

c) Asimiladores.- Si usan la conceptualización abstracta y la observación reflexiva.

d) Acomodadores.- $\mathrm{Si}$ se basan en la experiencia concreta y la experimentación activa.
Honey y Mumford (1986), a partir de la teoría de Kolb, desarrollaron los siguientes cuatro estilos de aprendizaje:

a) Activo.- Incluye a las personas activas que se involucran con las experiencias nuevas, se dejan llevar por los acontecimientos. Tienden a actuar primero y pensar después en las consecuencias.

b) Reflexivo.- Incluye a las personas que son observadoras y analizan sus experiencias desde diferentes perspectivas. Tratan de recoger datos $\mathrm{y}$ analizarlos detalladamente antes de llegar a una conclusión.

c) Teórico.- Corresponde a las personas que adaptan e integran sus observaciones en teorías complejas y bien fundamentadas lógicamente. Analizan y sintetizan la información y su sistema de valores prioriza la lógica y la racionalidad.

d) Pragmático.- Incluye a las personas que prueban ideas, teorías y técnicas nuevas, y tratan de comprobar si funcionan en la práctica. Les desagradan las largas discusiones sobre un mismo tema. Son prácticos y apegados a la realidad.

Estos estilos, de acuerdo con el modelo teórico de Honey y Mumford, fueron modificados en su contenido por Alonso, Gallego y Honey (1994, 2002), quienes los redefinen como:

a) Activo.- Corresponde a las personas que se caracterizan por ser animado- 
ras, improvisadoras, descubridoras, espontáneas y arriesgadas. Están interesadas en vivir las experiencias y ser cambiantes.

b) Reflexivo.- Incluye a las personas que son ponderadas, receptivas, analíticas y exhaustivas. Son observadoras, pacientes, detallistas, investigadoras y asimiladoras.

c) Teórico.- Caracteriza a las personas que son metódicas, lógicas, objetivas, críticas y estructuradas; son disciplinadas, ordenadas, buscadoras de hipótesis y teorías, además de exploradoras.

d) Pragmático.- Incluye a las personas experimentadoras, prácticas, eficaces y realistas; se caracterizan por ser rápidas, organizadoras, estar seguras de sí mismas, de solucionar problemas y de planificar sus acciones.

\section{La Teoría Clásica de los Tests (TCT)}

Se inicia con los trabajos de Binet, quien en 1907 construyó el primer test estandarizado de inteligencia, teniendo como marco de desarrollo los trabajos de Galton, Wundt, Ebbinghauss, Pearson y Spearman, los cuales enmarcaron el desarrollo de la Antropometría y la Psicofísica (Aiken, 1996). El aporte esencial de Binet fue la estandarización, las pruebas con múltiples ítems y el uso de normas, que constituyen las bases de la TCT, la cual se desarrollaba bajo el paradigma experimental con la manipulación y el control de variables (Anastasi \& Urbina, 1998).

Se asume que los puntajes de los tests expresan la habilidad y que existen factores en el medio ambiente que pueden alterarlos $\mathrm{y}$, por tanto, deben ser controlados en el proceso de aplicación de los tests. Spearman formaliza esta concepción como un modelo lineal simple, con una puntuación verdadera y un puntaje de error; uniéndose las ideas de normas de los tests y la población de ítems en el modelo probabilístico basado en el modelo de la TCT (Cortada, 1999; Brown, 1993).

En términos históricos, en 1950, Gulliksen, en su libro Theory of mental tests, presenta un desarrollo coherente de los principales conceptos de la teoría y expone los resultados de la confiabilidad (Cortada, 2000).

En 1954, la APA publicó las primeras recomendaciones técnicas para los test psicológicos y las técnicas de diagnóstico (APA, 1986). También en 1954, Meehl publicó los resultados de su investigación, en la que demostraba que la predicción estadística de los tests era más efectiva que el juicio clínico (Martínez, 2006).

En 1966, Novick, en su artículo "The axioms and principal results of classical test theory", sustenta la teoría sobre una base axiomática más rigurosa; y en 1968, Lord y Novick, en su libro Statistical theories of mental test scores, sientan las bases del desarrollo 
del enfoque de la Estructura Latente (Lord \& Novick, 1974) y que al decir de Van Den Wollenberg es el fin de la era de la TCT y el inicio de una nueva etapa para construir pruebas (Muñiz, 1994). El modelo se representa por la siguiente ecuación:

$$
X_{i}=T+E_{i}
$$

Donde:

$\mathrm{Xi}=$ Puntuación observada.

$\mathrm{T}=$ Puntuación verdadera constante.

Ei $=$ Puntuación de error.

Los principales supuestos teóricos de la Teoría Clásica de los Tests (Allen \& Yen, 2002; Muñiz, 1996, 1994) son los siguientes:

a) La expectativa del promedio de los errores es 0 , lo que indica que en una muestra grande de puntajes los errores tienden a suprimirse.

b) La correlación de la puntuación verdadera de un ítem con su error es 0 .

c) La correlación de la puntuación verdadera de un ítem con el error de otro ítem es 0 .

d) La correlación entre los errores de 2 ítems diferentes es 0 .

De los supuestos presentados se derivan las siguientes conclusiones:

a) El valor esperado de las puntuaciones obtenidas es igual al valor esperado teóricamente.

b) La varianza de las puntuaciones observadas es la suma de las varianzas de las puntuaciones verdaderas más el error.

c) Se asume que cada ítem es igualmente un indicador adecuado del mismo puntaje verdadero.

Este modelo desarrolló el estudio de la confiabilidad, que puede ser definida como el grado de consistencia que existe entre dos medidas de un mismo evento, objeto o constructo (Cortada, 2000; Anastasi \& Urbina, 1998; Muñiz, 1996; Nunnally \& Bernstein, 1995; Muñiz, 1994; Brown, 1993; Kline, 1993; Magnusson, 1976). El modelo de la TCT ha permanecido durante más de 70 años como el método más utilizado para construir instrumentos psicológicos, pero con el desarrollo de métodos alternativos, las cosas han cambiado en la actualidad (Muñiz, 1997; Anastasi \& Urbina, 1998; Cortada, 2000).

\section{La Teoría de la Respuesta al Ítem (TRI)}

Esta teoría parte del supuesto de que las puntuaciones obtenidas en un ítem $-\mathrm{y}$ por ende en un test- dependen directamente del grado en que la persona posea la habilidad o rasgo medido. Este rasgo no es observado, por ello se denomina rasgo latente. La relación que existe entre el rendimiento (puntuaciones observables) y el rasgo latente (habilidad de la persona) se describe a través de una función matemática. El rasgo latente es un constructo que permite explicar los hechos. 
Andrich (1988) y Escurra \& Delgado (1994) indican que los modelos de estructura latente en general se caracterizan por asumir que cuando un ítem mide un determinado rasgo latente, las respuestas de las personas ante dicho ítem deben presentar un gráfico o curva característica del ítem (CCI).

Esta teoría se origina cuando los investigadores en el campo de la psicometría identifican algunas limitaciones de la Teoría Clásica de los Tests (TCT), la cual ha presentado serias deficiencias que han sido superadas en el campo de la psicometría moderna por la Teoría de la Respuesta al Ítem (Delgado, Escurra \& Torres, 2006; Du Toit, 2003; Allen \& Yen, 2002; Bond \& Fox, 2001; Embretson y Reise, 2000; Van Der Linden \& Hambleton, 1997; Muñiz, 1997, 1996, 1996a; López Pina, 1995; Hambleton, Swaminathan \& Rogers, 1991). Las principales deficiencias identificadas en la TCT son las siguientes:

a) Los resultados de los instrumentos que miden diferentes constructos no pueden ser directamente comparables entre sí.

b) Los instrumentos dependen tanto de las muestras con las que se han trabajado como de la muestra de los ítems que conforman el test.

Por su parte, en contraposición, la TRI presenta las siguientes características básicas: a) Invarianza del parámetro de la habilidad: implica que la estimación de la habilidad de una persona es independiente de la muestra de ítems que conforman el test.

b) Invarianza de los parámetros del ítem: implica que las características psicométricas de un test, como la dificultad y la discriminación, son independientes de la muestra específica utilizada para estudiar las características del ítem.

c) Cálculo de un estadístico que indica la precisión con la que se estima la habilidad o rasgo de cada sujeto, por lo que puede variar de un sujeto a otro.

Las principales etapas históricas en el desarrollo de la TRI, de acuerdo con Van Der Linden \& Hambleton (1997), son las siguientes:

- En 1936, Richardson proporcionó las primeras vías para obtener estimaciones de los parámetros de la Teoría del Rasgo Latente, al derivar relaciones entre los parámetros obtenidos con la TCT, por una parte, y la Teoría del Rasgo Latente, por otra; sin embargo, el origen de la TRI, como tal, se atribuye a Ferguson y Lawley (Andrich, 1988).

- En 1952, a raíz de su publicación sobre las puntuaciones de los tests, Lord es considerado el padre de la TRI, aunque en principio este mis- 
mo autor la llamó la Teoría de la Curva Característica del Ítem. En esa misma publicación Lord describió el modelo de la Ojiva Normal de 2 parámetros.

- En 1960, Rasch publicó su libro sobre los Modelos Probabilísticos para Tests de Inteligencia y Atención, desarrollando los 3 principales modelos que constituyen la Teoría del Rasgo Latente.

- En 1968, Lord y Novick dedicaron cinco capítulos de su libro a esta teoría, desarrollando de manera matemática los modelos de 2 y 3 parámetros (Lord \& Novick, 1974).

- En 1969, Wright y Panchapakesan diseñan el programa Bical.

- En 1971, Mokken escribio el libro donde expone su teoría para construir escalas de medición.

- En 1974, Lord escribió el programa Logist.

- En 1977, Lord cambió el nombre a su modelo conocido como Teoría de la Curva Característica del Ítem por el de Teoría de Respuesta al Ítem.

- En 1979, Wright y Stone describieron en profundidad el modelo de un parámetro de Rasch.

- En 1980, Lord publica una revisión actualizada de desarrollo teórico y las aplicaciones del modelo de 3 parámetros.

- En 1982, Lord publica la segunda edición del programa Logist.

\section{El Modelo del Parámetro Logist de} Rasch

Este modelo fue desarrollado por el matemático danés George Rasch. Considera que la probabilidad de contestar correctamente un ítem está en función de su dificultad y de la habilidad de la persona.

Se caracteriza por estudiar únicamente el parámetro de la dificultad, mientras se asumen como constantes a los demás parámetros (De Ayala, 2009; Elorza, 2007; Wilson, 2005; Tristán, 2001; Embretson \& Reise, 2000; García Cueto, 1993).

Esta propuesta ha sido relacionada con los procedimientos utilizados para construir las escalas Guttman, en las cuales los ítems son seleccionados ordenadamente según su nivel de dificultad, de tal manera que cualquier sujeto que falle en el ítem $\mathrm{X}$ deberá fallar todos los ítems más difíciles que $\mathrm{X}$ y acertar todos los ítems más fáciles que X (Andrich, 1988).

A continuación se muestran el gráfico y la fórmula que lo representa: 


\section{Gráfico 1}

\section{Curva característica del ítem (CCl) del Modelo de Rasch}

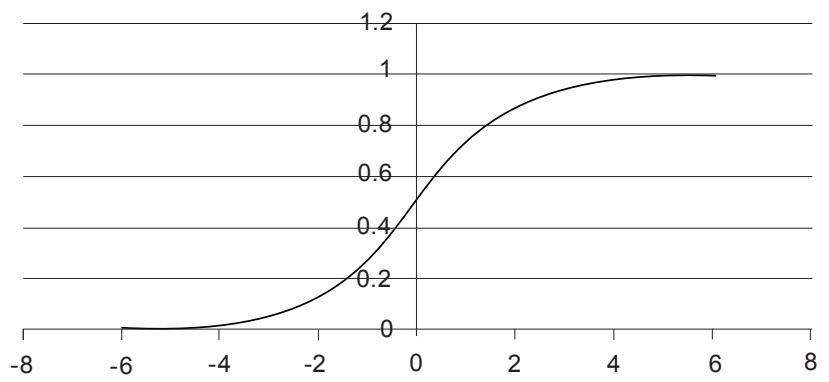

La fórmula generalizada del modelo de Rasch es la siguiente:

$$
P i(w)=\frac{e^{D a(W-b i)}}{1+e^{D a(W-b i)}}
$$

Siendo:

a: Índice de discriminación constante

bi: Dificultad del ítem

D: Valor escalar equivalente a 1.7

e: Base de los logaritmos naturales (2.718281828)

W: Nivel de habilidad.

La aplicación del modelo matemático presenta cinco axiomas, los cuales pueden ser demostrados empíricamente (Muñiz, 1997, 1996, 1990; Escurra \& Delgado, 1994; Hambleton, Swaminathan \& Rogers, 1991), y que corresponden a:

a) Dicotomía de los items.- La variable medida debe ser presentada de forma binaria, ya sea ( $\mathrm{V}$ o F), acierto o error, aunque es posible generalizar el modelo a ítems politómicos.

b) Monotonicidad.- La probabilidad de presentar la respuesta correcta debe incrementarse a medida que aumentan los niveles de la habilidad de los sujetos para todos los ítems.

c) Suficiencia estadística de la simple suma.- Toda la información necesaria para calcular los niveles de dificultad de los ítems y los niveles de habilidad de las personas pueden ser calculados a partir del número de respuestas correctas.

d) Unidimensionalidad.- Los ítems deben ser homogéneos, esto es, deben medir un solo rasgo latente (constructo) común a todos ellos. Para Hambleton, Swaminathan y Rogers (1991), un test es unidimensional cuando las distribuciones de los puntajes del test en todos los niveles de habilidad y en todas las subpoblaciones establecidas según niveles de ha- 
bilidad son idénticas. Se recomienda que este supuesto sea comprobado a través de procedimientos estadísticos específicos.

e) Independencia local estocástica.Indica que la única fuente de asociación entre los ítems es el rasgo latente, el cual es fijo, mientras que las respuestas a los ítems deben ser independientes entre sí. Estos pueden ser estudiados a través de diferentes procedimientos estadísticos.

Cuando todos los supuestos son verificados de forma empírica, es posible afirmar que se cumple la especificidad objetiva, lo cual implica que para el universo de sujetos y de medidas, la selección de los ítems es irrelevante para la medida de los sujetos, y la selección de estos es irrelevante para la medida de los ítems (Escurra \& Delgado, 1994). Esto permite concluir que la aplicación del muestreo aleatorio para la selección de los sujetos durante el proceso de construcción de pruebas se torna irrelevante, aunque se hace necesaria la comparación de diversos tipos de muestras para analizar las evidencias de validez y generalidad del modelo estudiado. Es debido a esta propiedad que se pueden obtener:

a) Pruebas que miden diferentes constructos cuyos resultados pueden ser directamente comparables entre sí.

b) Instrumentos universales construidos a partir de muestras representativas de las poblaciones. c) Instrumentos con unidades de medidas que pueden ser catalogadas por lo menos como de intervalo.

Los modelos matemáticos permiten expresar de forma numérica y también gráfica la relación entre el rasgo latente y la posibilidad de emitir la respuesta correcta a un ítem. Inicialmente se utilizó en su construcción la ojiva normal, $\mathrm{y}$ actualmente estos modelos han sido reemplazados por los modelos logísticos, debido a que en términos computacionales son más simples.

\section{Investigaciones antecedentes}

Con respecto a los estudios realizados sobre la comparación del modelo de la TCT con la TRI, la bibliografía reporta que:

a) Adedoyin, Nenty y Chilisa (2008) estudiaron la invarianza de los parámetros de dificultad de los ítems de acuerdo con la TCT y la TRI. Ellos trabajaron con una muestra de 35.262 estudiantes de escuelas secundarias de Botswana, seleccionando las muestras de acuerdo con el género, las regiones educacionales y el nivel de habilidad de los alumnos. El instrumento utilizado fue una prueba de matemáticas para alumnos de secundaria. Se estimaron los parámetros de dificultad de acuerdo con los algoritmos de cada uno de los modelos psicométricos evaluados. Los resultados indicaron que los parámetros de dificultad 
calculados con la TCT variaron de acuerdo con las diferentes muestras independientes comparadas, en tanto que los parámetros calculados con la TRI según el modelo de Rasch, fueron invariantes al compararse en dichas muestras. Los autores concluyeron que el modelo psicométrico de la TCT produce estimaciones inestables del parámetro de dificultad de los instrumentos, mientras que la TRI sí produce estimaciones del parámetro de dificultad invariante.

b) Prieto, Alonso y Lamarca (2003) compararon los modelos de la TCT y la TRI para desarrollar una forma corta del Cuestionario de Calidad de Vida, el cual estaba conformado por 38 ítems y fue aplicado a una muestra de 9419 participantes de diferentes ciudades de España, quienes tenían entre 12 y 78 años de edad, siendo el $52,1 \%$ mujeres y el $47,5 \%$ varones. La aplicación de la metodología de la TCT a la prueba original permitió desarrollar una versión conformada por 20 ítems y 4 dimensiones, y cuando se aplicó la TRI, de acuerdo con el modelo de Rasch, se conformó una versión de 22 ítems y 2 dimensiones. Al compararse ambas versiones, los resultados indicaron que una versión más estable y consistente de la prueba original fue la versión de 22 ítems desarrolladas con la TRI.

c) Prieto y Delgado (2003) construyeron una prueba de matemáticas que está conformada por 30 ítems. El instrumento fue aplicado a una muestra de 455 alumnos de secundaria provenientes de colegios de Salamanca (España), de los cuales 241 fueron varones y 214 mujeres. Los datos fueron analizados aplicando el modelo de Rasch; los resultados permitieron corroborar que los ítems se ajustaban a la exigencia del modelo utilizado; por ello, los autores recomiendan la aplicación del instrumento tanto en la evaluación colectiva como en el diagnóstico individual.

d) Jackson, Draugalis, Slack, Zachry y D'Agostino (2002) desarrollaron un estudio en el cual se construyó y validó un test para evaluar los resultados del pensamiento crítico relacionados con la evaluación de la literatura científica en el Doctorado en Farmacia en la Universidad de Arizona. Se desarrollaron 25 ítems de acuerdo con la taxonomía jerárquica de Bloom. Los análisis psicométricos se realizaron de acuerdo con la TCT y la TRI con el modelo de Rasch. Los hallazgos permitieron concluir que el instrumento cumple con todos los requerimientos exigidos por el modelo de Rasch.

e) Fan (1998) realizó el análisis comparativo de la estimación de los parámetros del ítem y los parámetros de las personas de acuerdo con la TCT y la TRI, para ello se aplicó la prueba de Evaluación de las Habilidades 
Académicas de Texas a una muestra de 193.240 alumnos de secundaria, de los cuales el 50,8\% eran mujeres y el $49,2 \%$ varones. El instrumento estuvo conformado por 48 ítems de lectura y 60 ítems de matemáticas. Los resultados demostraron que tanto a nivel de los parámetros de los ítems como de las personas existió una ligera diferencia a favor del modelo de la TRI, aunque recomienda realizar más investigaciones.

f) Gutiérrez y Gonzales-Tablas (1992) realizaron el análisis de la calibración de los ítems del PMA-E bajo el modelo de Rasch. El instrumento estaba conformado por 120 ítems y fue aplicado a una muestra de 604 estudiantes de secundaria de la ciudad de Salamanca (España), de entre 13 y 16 años, 274 fueron mujeres y 330 , varones. El análisis del modelo de Rasch se realizó con el programa MicroCat, que incluye a los subsistemas Iteman, Rascal y Ascal. La estimación de los parámetros de dificultad de los ítems y los parámetros de habilidad de las personas demostraron que todos los ítems cumplieron con los requisitos exigidos por el modelo.

En relación con las investigaciones psicométricas realizadas con el Cuestionario de Honey y Alonso de Estilos de Aprendizaje (CHAEA), en la bibliografía se reporta que solo se han realizado estudios bajo el modelo de la TCT, entre los que destacan: a) Adán (2007) realizó una investigación para analizar las relaciones entre los estilos de aprendizaje, las modalidades de bachillerato y el rendimiento académico en estudiantes españoles. Para evaluar los estilos de aprendizaje se utilizó el CHAEA, que fue aplicado a una muestra de 556 alumnos del bachillerato de La Rioja, distribuidos en grupos en función de la modalidad y opciones cursadas. Los resultados indicaron que los alumnos con mejor rendimiento académico presentaron un perfil de aprendizaje caracterizado por obtener una mayor valoración en el estilo reflexivo y teórico y una menor puntuación en los estilos activo y pragmático.

b) Contreras (2007), trabajando con alumnos de Ingeniería y del área de negocios en la ciudad de México, aplicó el CHAEA, los hallazgos indicaron que predomina el estilo reflexivo en las mujeres y el pragmático en los hombres. La comparación por carrera de estudio indicó que predomina el estilo activo en el área de negocios y el pragmático en Ingeniería.

c) Gómez y Yacarini (2006) desarrollaron un estudio para identificar los estilos de aprendizaje que predominan en los estudiantes de primer año de la Universidad Católica Santo Toribio de Mogrovejo (USAT). Para ello se utilizó el Cuestionario de Honey y Alonso de Estilos de Aprendizaje 
(CHAEA). Los resultados alcanzados indicaron que el estilo de aprendizaje que más predomina es el de tipo reflexivo, seguido por el teórico, el pragmático y el activo.

d) Bustinza, Durán y Quintasi (2005) evaluaron los estilos de aprendizaje de estudiantes de la especialidad en Educación Inicial en el Perú; para ello utilizaron el CHAEA, encontrando que predominan los estilos activo y reflexivo. Al analizar la relación entre los estilos con el rendimiento, se apreció que en los alumnos con el rendimiento más bajo predomina el estilo activo.

e) Escurra (2005) realizó un estudio en el Perú utilizando el CHAEA junto con el Inventario de Estrategias de Aprendizaje y Estudio y el rendimiento académico en 1260 estudiantes universitarios. Los resultados indicaron que el Cuestionario de Honey y Alonso de Estilos de Aprendizaje presentó evidencias de validez de constructo y confiabilidad por consistencia interna. También se apreció que el estilo activo, el estilo reflexivo, las estrategias para el examen, las ayudas para el estudio, la administración del tiempo y la autoevaluación influyen de forma positiva sobre el rendimiento académico de los alumnos universitarios. Adicionalmente se observaron diferencias por sexo y tipo de universidad. f) Luengo y González (2005) aplicaron el CHAEA a los alumnos de tercero de secundaria en España. Los hallazgos indicaron que entre los alumnos predominan los estilos activo y pragmático. También se apreció que el rendimiento en matemáticas está asociado con el predominio de los estilos teórico y reflexivo. Adicionalmente se pudo apreciar una fuerte relación entre los estilos activo y pragmático, y una menor relación en los estilos teórico y reflexivo.

g) Gómez del Valle et al. (2003), por su parte, realizaron un estudio para determinar los estilos de aprendizaje predominantes entre los alumnos de Magisterio de la Facultad de Ciencias de la Educación de la Universidad de Cádiz. Para ello aplicaron el CHAEA a una muestra de 153 participantes, de los cuales 54 fueron del primer año, 53 del segundo año y 46 del tercer año. Los resultados permitieron concluir que no existieron diferencias estadísticas significativas entre los grupos de alumnos en sus estilos de aprendizaje.

h) Ordóñez, Rosety Rodríguez y Rosety Plaza (2003) realizaron un estudio en los alumnos de Ciencias de la Salud en la Universidad de Cádiz, aplicando el CHAEA para evaluar los estilos de aprendizaje. Los resultados permitieron apreciar que predominan los estilos reflexivo y pragmático. 
i) Camarero, Martín del Buey y Herrero (2000) aplicaron el CHAEA a una muestra de 447 alumnos de los niveles iniciales y finales de cinco carreras en la Universidad de Oviedo. Los resultados permitieron indicar que los estilos teórico y reflexivo caracterizan a los estudiantes universitarios independientemente de sus carreras. Mientras los alumnos de Educación y Derecho utilizan más el estilo activo, los alumnos de Informática y Matemáticas e Informática y Derecho utilizan más el estilo pragmático. Finalmente, en los alumnos de Física se encontró nivel medio en dichos estilos.

\section{Objetivos}

Se plantearon los siguientes objetivos:

a) Identificar las principales características psicométricas del Cuestionario de Honey y Alonso de Estilos de Aprendizaje (CHAEA), bajo el modelo de la Teoría Clásica de los Tests.

b) Establecer las principales características psicométricas del Cuestionario de Honey y Alonso de Estilos de Aprendizaje (CHAEA), bajo el modelo de Rasch de la Teoría de la respuesta al ítem.

c) Comparar el desempeño de los modelos psicométricos de la TCT y la TRI bajo el Modelo de Rasch, al ser aplicados al Cuestionario de Honey y Alonso de Estilos de Aprendizaje
(CHAEA) en estudiantes universitarios de la ciudad de Lima.

\section{MÉTODO}

La presente investigación, de acuerdo con Kerlinger y Lee (2002), corresponde a un estudio de tipo metodológico, en la medida en que se trata de evaluar cuál de los modelos de medición es más efectivo.

De acuerdo con lo que proponen Hernández, Fernández y Baptista (2006) y Sánchez y Reyes (2006), el estudio puede ser clasificado como aplicado, en la media en que se orienta a la aplicación, de manera práctica, de los conocimientos teóricos de un determinado tema, que en este caso corresponde a la revisión de las propiedades psicométricas de las pruebas psicológicas. Adicionalmente, para Alarcón (2008) el estudio se puede clasificar como una investigación psicométrica.

\section{Población y muestra de estudio}

Para el desarrollo del estudio se reanalizaron los datos obtenidos en la investigación "Estilos, estrategias de aprendizaje y rendimiento académico en estudiantes universitarios de Lima metropolitana" (PI. 50.006.2005), efectuada el año 2005 con los auspicios del Instituto de Investigación Científica de la Universidad de Lima.

La muestra estuvo conformada por 1260 alumnos universitarios de Lima 
metropolitana, de los cuales el 38,4\% provenían de universidades con gestión no estatal y el $61,6 \%$ con gestión estatal. En relación con el género se observó que el 52,1\% fueron varones en tanto que el $47,9 \%$ fueron mujeres.

El análisis de la muestra, tomando en cuenta la universidad de procedencia y el género, permitió apreciar que los varones de la universidad nacional constituyeron el $33,9 \%$ y las mujeres el $27,7 \%$, en tanto que en la universidad no estatal los varones conformaron el $18,2 \%$ y las mujeres el $20,2 \%$.

En lo que respecta a las principales características de la muestra de alumnos se encuentra que estudió Ciencias de la Salud (Medicina Humana, Veterinaria, Odontología y Psicología) el 26,4\%; Ciencias Exactas (Matemáticas, Química, Estadísticas) el 19,2\%; Ingeniería (Civil, Mecánica, Informática) el 16,8\%; Ciencias de la Administración (Contabilidad, Economía, Administración) el 18,3\%; y Humanidades (Educación, Derecho, Sociología) el 19,3\% de la muestra. Respecto de la edad, los hallazgos indicaron que los alumnos tenían entre 18 y 30 años, siendo la mayor proporción la correspondiente al grupo de 20 a 25 años $(57,8 \%)$. Con relación al lugar de procedencia, la gran mayoría era de Lima $(85,6 \%)$ en tanto que el $14,4 \%$ era de provincias. También se apreció que el 95,2\% eran solteros(as) mientras que el $4,8 \%$ eran casados(as).
Instrumento de recolección de datos. Cuestionario de Honey y Alonso de Estilos de Aprendizaje (CHAEA)

El cuestionario mide el estilo de aprendizaje de acuerdo con los aportes teóricos de Kolb (1984) y Honey y Mumford (1986), quienes coinciden en la definición y desarrollo del proceso de aprendizaje, que sería un proceso cíclico dividido en cuatro etapas, en las que influiría en un alto porcentaje las experiencias vividas, las circunstancias medioambientales y lo heredado.

El instrumento está conformado por 80 enunciados presentados en forma de preguntas, de las cuales 20 corresponden a cada uno de los siguientes estilos de aprendizaje:

- Activo

- Reflexivo

- Teórico

- Pragmático

El sistema de calificación indica que se debe responder de forma dicotómica: De acuerdo o En desacuerdo. Se suman las respuestas afirmativas y se obtiene para cada estilo el puntaje total, el cual se compara con los baremos respectivos.

Como reportan Alonso, Gallego y Honey (2002, 1994), el instrumento presenta validez y confiabilidad, estudiadas originalmente en una muestra de 1371 alumnos de 25 facultades de las universidades Autónoma y Politécnica de Madrid. 
El estudio realizado por Escurra (2005) permitió corroborar que el instrumento cumplía con las propiedades psicométricas de evidencias de validez y confiabilidad en los estudiantes peruanos.

Técnicas de procesamiento y análisis de datos

De acuerdo con la bibliografía especializada (De Ayala, 2009; Santisteban, 2009; Furr \& Bacharach, 2008; Montesinos, 2008; Linacre, 2007; Bond \& Fox, 2001; Embretson \& Reise, 2000; Van Der Linden \& Hambleton, 1997; Muñiz, 1997, 1996, 1996a; Kline, 1993; Hambleton, Swaminathan \& Rogers, 1991) se realizarán los siguientes tipos de análisis estadísticos:

I. Análisis psicométrico con el modelo de la Teoría Clásica de los Tests; en cada uno de los estilos se calcularán:

a) Correlaciones ítem-tests corregidas, para determinar la consistencia interna entre el ítem y el puntaje total.

b) Coeficiente Alfa de Cronbach, para determinar la confiabilidad de los ítems que conforman la escala evaluada.

c) Análisis factorial confirmatorio, para comprobar si existe una determinada estructura factorial de los ítems en nuestro medio.

II. Análisis psicométrico con el Modelo de Rasch de la Teoría de la Respuesta al Ítem, para lo cual se utilizará un programa específico como, por ejemplo, el programa Winsteps (Linacre, 2007):

a) Análisis gráfico de las curvas características de los ítems con el fin de establecer la amplitud con la que se mide el constructo evaluado y el análisis del test de información, para establecer el intervalo de mayor precisión respecto al nivel de la habilidad o constructo evaluado.

b) Calibración de los ítems en lo que respecta a los parámetros de dificultad del ítem con sus respectivos índices de bondad de ajuste:

b.1 Infit (Information-pattern sensitive fit statistics), que evalúa el ajuste interno, pues es sensible al comportamiento inesperado que afecta a los ítems cuya dificultad está cerca del nivel de habilidad medida de las personas (Bond \& Fox, 2001). Se incluirán los análisis de la MNSQ (media cuadrática del ajuste interno), la cual debe alcanzar valores entre 0.50 y 1.30 para ser aceptable y el ZSTD (media cuadrática del ajuste interno estandarizado). Se establecerá como criterio de rechazo si alcanza un valor mayor de 2 o menor de -2 . 
b.2 Outfit (Outlier-sensitive fit statistics), que evalúa el ajuste externo y es sensible al comportamiento inesperado que afecta a los ítems cuya dificultad está lejos del nivel de habilidad medida de las personas (Bond \& Fox, 2001). Se incluirán los análisis de la MNSQ (media cuadrática del ajuste externo), la cual debe alcanzar valores entre 0.50 y 1.30 para ser aceptable y el ZSTD (media cuadrática del ajuste externo estandarizado). Se establecerá como criterio de rechazo si alcanza un valor mayor de 2 o menor de -2 .

c. Evaluación de la unidimensionalidad del modelo, que utilizará la estrategia propuesta por Smith y Miao (1994), para lo cual se realizará el análisis de los componentes principales de los residuos después de extraerse el Modelo de Rasch, de manera que los factores adicionales con eigenvalues mayores de 1.4 se consideran violaciones de la unidimensionalidad. Luego se seleccionarán los ítems de los factores positivo y negativo con saturaciones mayores de 0.19 y menores de -0.19 , respectivamente, siendo cada grupo de ítems sometido al Modelo de Rasch de forma separada, correlacionándose las pun- tuaciones de los factores positivos con los del factor negativo. Para interpretar las correlaciones encontradas entre los factores extraídos de los residuos se asumirá la hipótesis de que se trata de tests paralelos y se considerará como valor límite $\mathrm{r}=0.51$ (Wright, 2000).

\section{Resultados}

Análisis psicométrico con la Teoría Clásica de los Tests del Cuestionario Honey y Alonso de Estilos de Aprendizaje (CHAEA)

El análisis de la confiabilidad de los estilos de aprendizaje permite apreciar en la tabla 1 que los coeficientes KuderRichardson 20 fluctúan entre 0.78 para el aprendizaje Teórico y 0.83 para el aprendizaje Activo. Además, se encuentra que los intervalos de las correlaciones item-test corregidas alcanzan valores superiores a 0.20 , con lo cual superan el criterio de aceptación propuesto por Kline (1986). Estos hallazgos corroboran que el CHAEA cumple con el requisito de la TCT de permitir obtener puntajes confiables.

Adicionalmente, en la tabla se encuentra que los resultados indican que en los alumnos predominan los estilos de aprendizaje Reflexivo ( $\mathrm{M}=14.74$ D. $\mathrm{E}:=3.20)$ y Teórico $(\mathrm{M}=14.21$ D. $\mathrm{E}:=3.09$ ). 
Tabla 1

Resumen del análisis de la confiabilidad de los estilos de aprendizaje del CHAEA

\begin{tabular}{lcccc}
\hline Estilo de aprendizaje & $\mathbf{M}$ & D. E. & Intervalo de ritc & Kr20 \\
\hline Activo & 12.13 & 3.53 & $0.20^{*}-0.50 *$ & 0.83 * \\
Reflexivo & 14.74 & 3.20 & $0.25^{*}-0.50 *$ & 0.80 * \\
Teórico & 14.21 & 3.09 & $0.22 *-0.64 *$ & 0.78 * \\
Pragmático & 12.21 & 3.58 & $0.20 *-0.71 *$ & 0.79 * \\
\hline
\end{tabular}

$N=1260$

El análisis de la validez del instrumento se realizó con el estudio de las evidencias de la validez de Constructo (Lissitz, 2009; Santisteban, 2009; Furr \& Bacharach, 2008), en su modalidad del método intraprueba a través del Análisis Factorial Confirmatorio, aplicándose el programa Amos 16.0 (Arbuckle, 2007). Los resultados obtenidos con el modelo teórico propuesto de un factor, de acuerdo con las recomendaciones propuestas por Blunch (2008), Thompson (2008), Brown (2006), Hancock y Mueller (2006), Schumacker y Lomas (2004), Byrne (2001) y por Raykov y Marcoulides (2000), se comparó con un modelo alternativo en el cual se asume que todos los factores son independientes.

Los resultados del Análisis Factorial Confirmatorio se incluyen en la tabla 2 y el gráfico 2; permiten observar que el modelo teórico de un factor que integra los estilos de aprendizaje presenta en el test de Bondad de Ajuste Chi-Cuadrado mínimo un valor de 2.83, el cual, con 1 grado de libertad alcanza una probabilidad de .092, indica que el modelo es adecuado.

Tabla 2

Análisis de la evidencia de la validez de constructo del CHAEA a través del Análisis Factorial Confirmatorio

\begin{tabular}{lcc}
\hline Datos & Modelo de un factor & Modelo independiente \\
\hline Parámetros & 9 & 4 \\
Chi-Cuadrado mínimo & 2.83 & 2507.99 \\
Grados de libertad (G. L.) & 1 & 6 \\
P & .092 & .000 \\
Chi-Cuadrado mínimo/G. L. & 2.83 & 417.99 \\
RMR & 0.24 & 3.99 \\
GFI & 0.99 & 0.61 \\
AGFI & 0.99 & 0.35 \\
\hline
\end{tabular}

$N=1260$ 
Gráfico 2

Modelo de ecuaciones estructurales de la evidencia de la validez de constructo del CHAEA

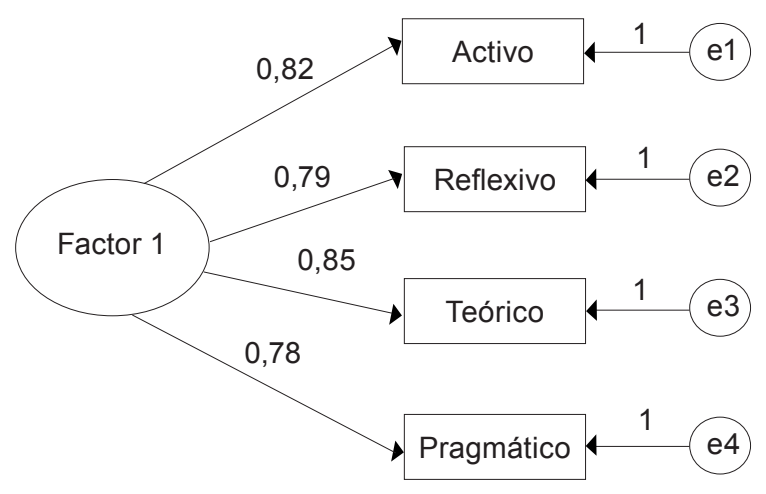

La revisión del Indice Residual de la Raíz Cuadrada Media de Residuales (RMR) que evalúa la aproximación de la matriz de covarianzas teórica con la matriz observada, presenta un valor pequeño ( $R M R=0.24)$ y los análisis complementarios de la Bondad de Ajuste a través del índice de Ajuste $(\mathrm{GFI}=0.99)$ y el índice de Ajuste Ponderado (AGFI $=0.99$ ) alcanzaron valores óptimos muy cercanos a 1. El conjunto de resultados alcanzados permiten aceptar el modelo de un factor, por lo que se concluye que el CHAEA presenta validez de constructo.

Análisis psicométrico con la Teoría de la Respuesta al Ítem del Estilo de Aprendizaje Activo del CHAEA

A continuación se presentan los resultados obtenidos en los ítems de los estilos de aprendizaje en cada una de las etapas del análisis bajo el modelo de Rasch.
Análisis gráfico de los ítems de los estilos de aprendizaje del CHAEA

El análisis de las curvas características de los ítems (CCI) del Estilo de Aprendizaje Activo, que incluye la medición conjunta de los puntajes del ítem y el nivel de habilidad que exhiben los sujetos (gráfico 3), muestra que el rango evaluado contiene desde los niveles logit muy bajos (-8) como en el ítem 27 , hasta niveles logit muy altos (7), como en el ítem 43. Este hallazgo indica que el estilo Activo mide, de forma amplia, el constructo evaluado.

El análisis del test de la función de información de los ítems del Estilo de Aprendizaje Activo permite identificar la precisión que presentan las mediciones realizadas con el instrumento evaluado. En el gráfico 4 se muestra que las mediciones más estables se pueden obtener en el caso de que la habilidad del 


\section{Gráfico 3}

\section{$\mathrm{CCl}$ de los ítems del Estilo de Aprendizaje Activo del CHAEA}

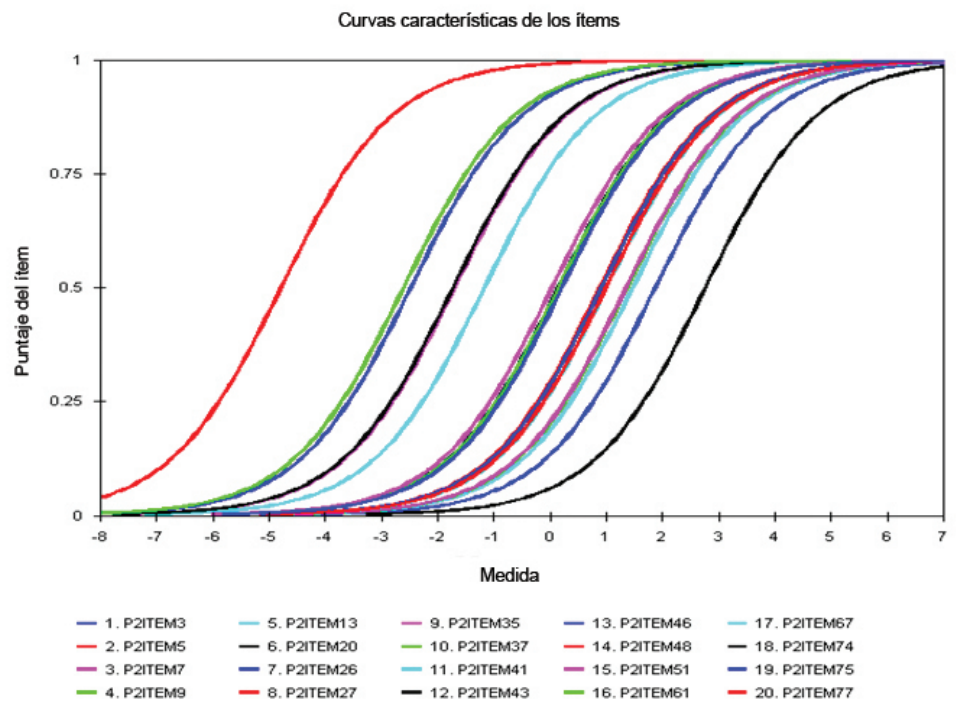

\section{Gráfico 4}

Función de información de los ítems del Estilo de Aprendizaje Activo del CHAEA

Función de información del test

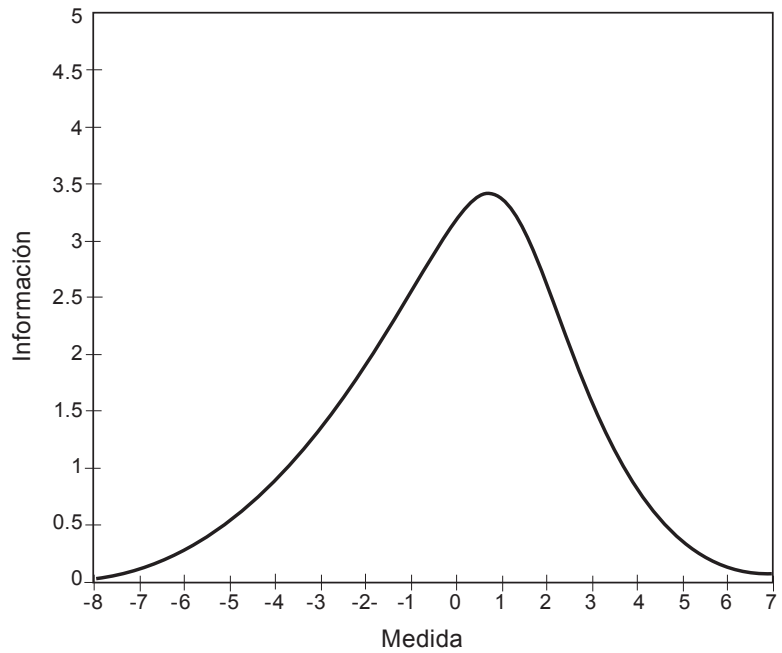


sujeto fluctúe entre -2 logit y 3 logit; si la habilidad presenta valores diferentes de los indicados, es decir valores más bajos o más altos, el resultado alcanzado es más impreciso y contiene mayor error, especialmente en los extremos.

Respecto al análisis de las CCI del Estilo de Aprendizaje Reflexivo, en el gráfico 5 se encuentra que el rango evaluado incluye desde los niveles logit muy bajos (-6), como es el caso del ítem 69, hasta niveles logit muy altos (7), como es el caso del ítem 79. Los hallazgos obtenidos permiten indicar que el estilo Reflexivo está conformado por ítems que miden de forma amplia el constructo evaluado.
En lo que concierne al análisis del test de la función de información de los ítems del Estilo de Aprendizaje Reflexivo, en el gráfico 6 se nota que las mediciones más estables se pueden obtener en el caso de que la habilidad del sujeto fluctúe entre -3 logit y 2 logit, de tal forma que cuando la habilidad presenta valores diferentes de los indicados, es decir valores más bajos o más altos el resultado que se alcanza tiende a ser más impreciso y presentar un mayor error de medición, especialmente en los extremos.

Respecto a la revisión de las CCI del Estilo de Aprendizaje Teórico, en el gráfico 7 se puede apreciar que el rango

\section{Gráfico 5}

\section{$\mathrm{CCl}$ de los ítems del Estilo de Aprendizaje Reflexivo del CHAEA}

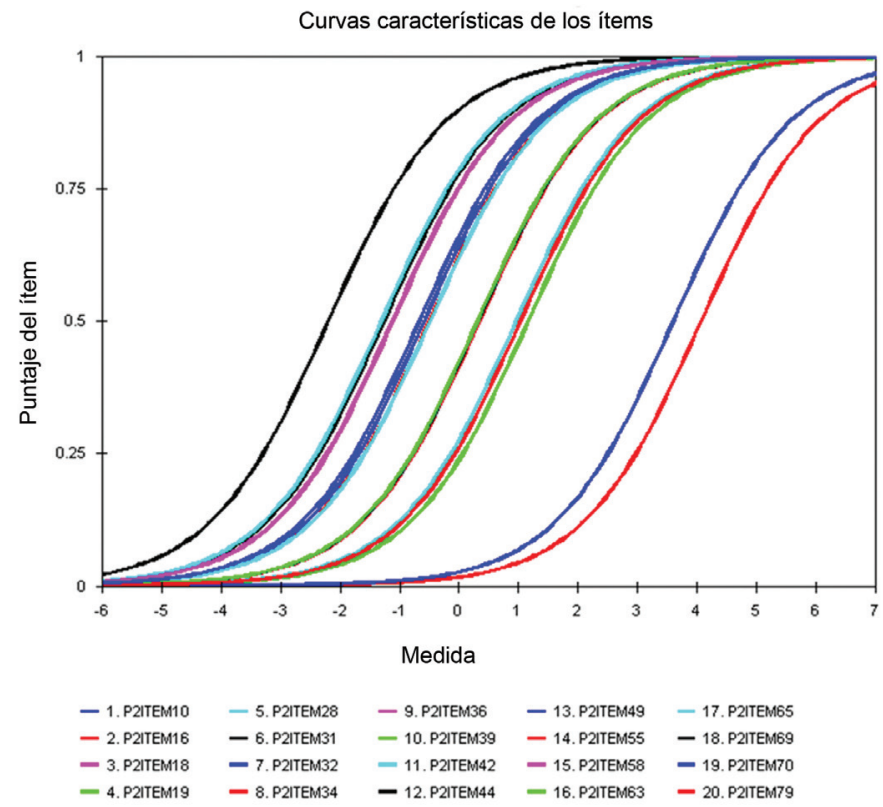


evaluado incluye desde los niveles $l o-$ git muy bajos (-8), como es el caso del ítem 54, hasta niveles logit muy altos (10), como es el caso del ítem 78. Los hallazgos alcanzados permiten indicar que el estilo Teórico incluye ítems que miden de forma amplia el constructo evaluado.

\section{Gráfico 6}

\section{Función de información de los ítems del Estilo de Aprendizaje Reflexivo del CHAEA}

Función de información del test

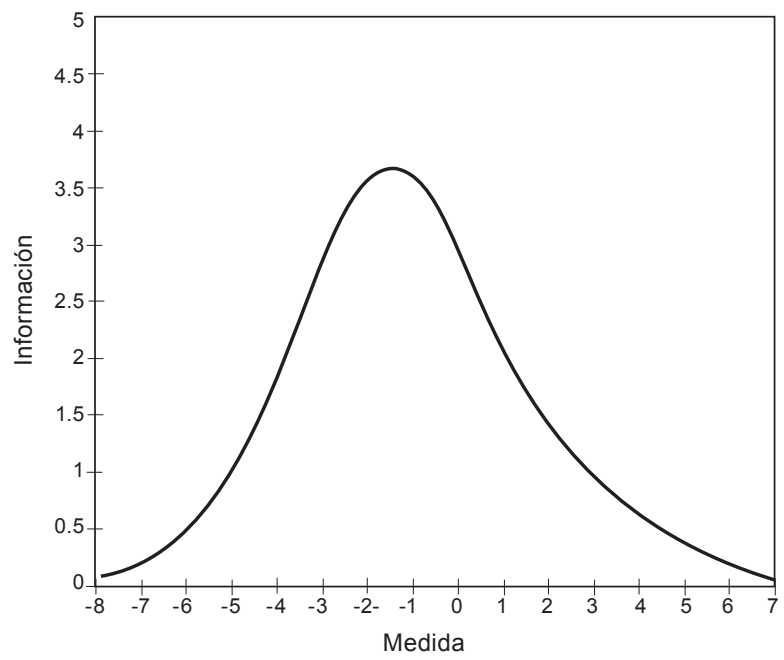

\section{Gráfico 7}

$\mathrm{CCl}$ de los ítems del Estilo de Aprendizaje Teórico del CHAEA

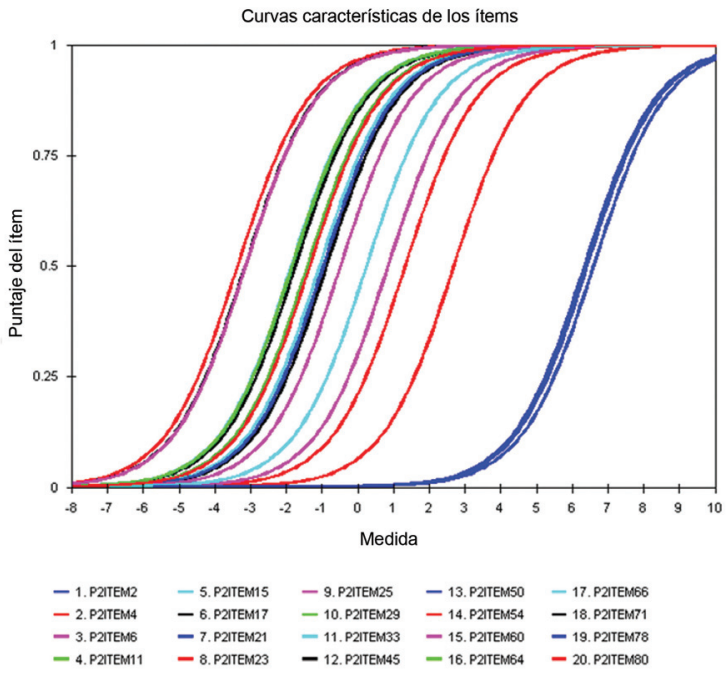


En el caso del análisis del test de la función de información de los ítems del Estilo de Aprendizaje Teórico, tal como se aprecia en el gráfico 8 , se encuentra que las mediciones más estables se pueden obtener en el caso de que la habilidad del sujeto fluctúe entre -4 logit y 1 logit, de tal manera que cuando la habilidad presenta valores diferentes de los indicados, es decir valores más bajos o más altos, el resultado que se alcanza tiende a ser más impreciso y contener un mayor error de medición, especialmente en los extremos.

En lo que concierne al análisis de las CCI del Estilo de Aprendizaje Prag- mático, en el gráfico 9 se encuentra que el rango evaluado incluye desde los niveles logit muy bajos (-8), como es el caso del ítem 22, hasta niveles logit muy altos (7), como el caso del ítem 1. Estos resultados permiten indicar que el estilo Pragmático está conformado por ítems que miden de forma amplia el constructo evaluado.

En relación con la revisión del test de la función de información de los ítems del Estilo de Aprendizaje Pragmático, en el gráfico 10 se puede notar que las mediciones más estables se pueden obtener en el caso de que la habilidad del sujeto fluctúe entre -3 logit y 3 logit,

\section{Gráfico 8}

Función de información de los ítems del Estilo de Aprendizaje Teórico del CHAEA

Función de información del test

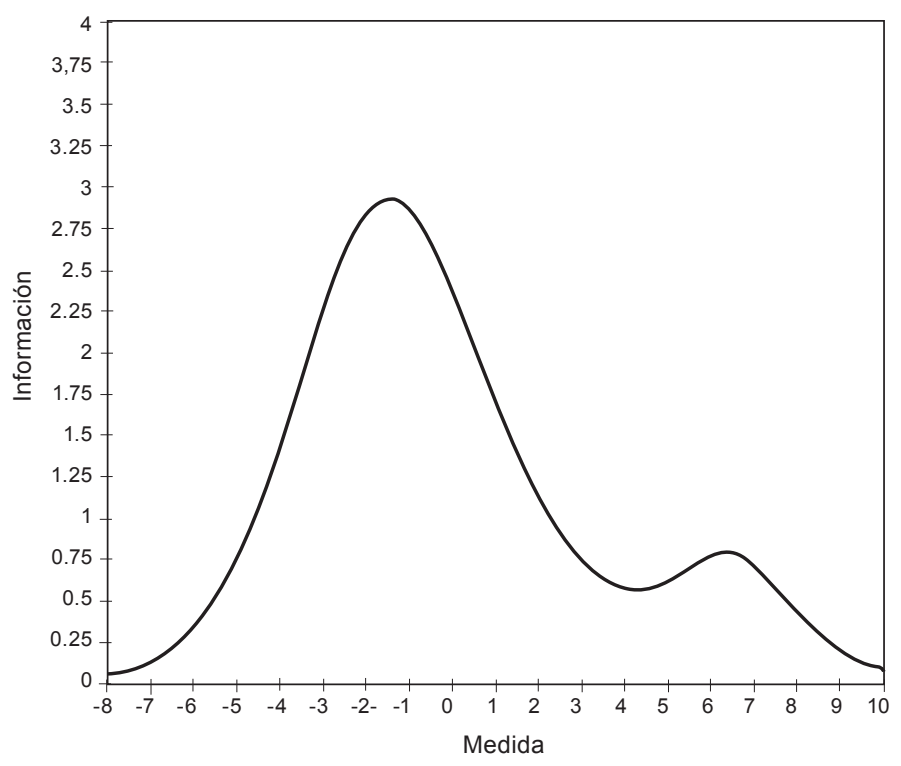




\section{Gráfico 9}

$\mathrm{CCl}$ de los ítems del Estilo de Aprendizaje Pragmático del CHAEA

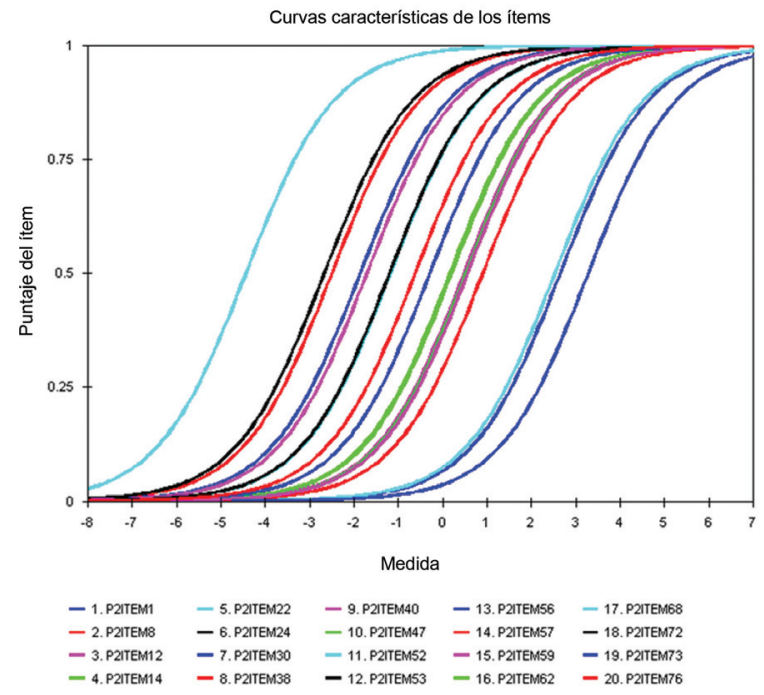

\section{Gráfico 10}

Función de información de los ítems del Estilo de Aprendizaje Pragmático del CHAEA

Función de información del test

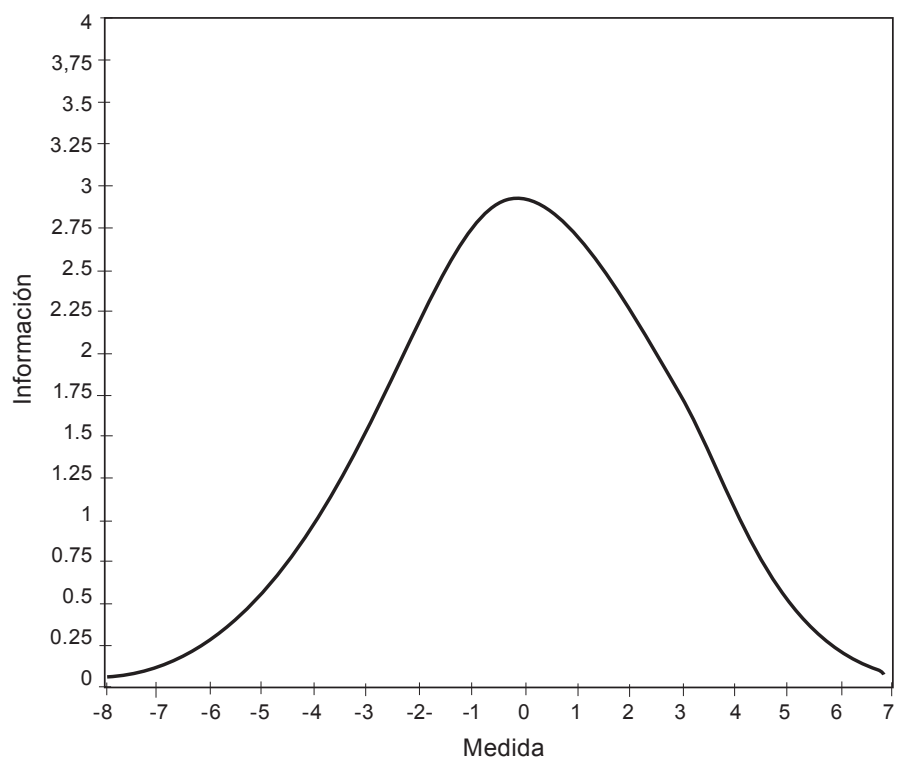


de tal manera que cuando la habilidad presenta valores diferentes de los indicados, ya sean valores más bajos o valores más altos, el resultado alcanzado es más impreciso y presenta más error, especialmente en los extremos.

Análisis de la calibración de los ítems

Los resultados del análisis psicométrico de la calibración de los ítems del Estilo de Aprendizaje Activo con el Modelo de Rasch (tabla 3), permiten apreciar que los parámetros del ítem (b) o difi- cultad, presentan valores que varían entre los niveles muy fáciles (-4.79), como es el caso del ítem 27, y los niveles muy difíciles (2.76), como es el caso del ítem 43 , es decir que existe un espectro relativamente grande de los niveles evaluados en el constructo. Adicionalmente, se observa que en la mayoría de ítems el error de estimación (E. E.) es pequeño. La revisión de los errores Infit, que evalúan el ajuste interno de los ítems, y de los Outfit, que evalúan el ajuste externo de los ítems, permite revelar que solamente los ítems 7 y 16 presentan

Tabla 3

Análisis de la calibración de los ítems del Estilo de Aprendizaje Activo del CHAEA

\begin{tabular}{|c|c|c|c|c|c|c|c|c|}
\hline \multirow{2}{*}{ Núm. } & \multirow{2}{*}{ Ítem } & \multirow{2}{*}{ b } & \multirow{2}{*}{ E. E. } & \multicolumn{2}{|c|}{ Infit } & \multicolumn{2}{|c|}{ Outfit } & \multirow{2}{*}{ rpbis } \\
\hline & & & & MNSQ & ZSTD & MNSQ & ZSTD & \\
\hline 12 & 43 & 2.76 & 0.09 & 1.23 & 4.40 & 2.97 & 9.90 & 0.19 \\
\hline 6 & 20 & -1.73 & 0.09 & 1.32 & 5.60 & 1.67 & 4.10 & 0.04 \\
\hline 17 & 67 & 1.47 & 0.07 & 1.45 & 9.90 & 1.32 & 5.40 & 0.29 \\
\hline 15 & 51 & -1.70 & 0.08 & 1.31 & 5.60 & 1.40 & 2.70 & 0.08 \\
\hline 19 & 75 & 1.87 & 0.07 & 1.05 & 1.10 & 1.38 & 5.20 & 0.46 \\
\hline 5 & 13 & 1.00 & 0.07 & 1.30 & 8.40 & 1.35 & 7.10 & 0.35 \\
\hline 2 & 5 & 0.99 & 0.07 & 1.05 & 1.50 & 1.30 & 6.30 & 0.47 \\
\hline 16 & 61 & 1.37 & 0.07 & 1.13 & 3.50 & 1.01 & 0.02 & 0.48 \\
\hline 7 & 26 & -2.48 & 0.11 & 1.12 & 1.40 & 0.92 & -0.04 & 0.15 \\
\hline 10 & 37 & 0.15 & 0.07 & 1.03 & 1.10 & 0.91 & -1.80 & 0.49 \\
\hline 8 & 27 & -4.79 & 0.10 & 0.98 & 0.10 & 0.37 & -2.70 & 0.12 \\
\hline 13 & 46 & 0.91 & 0.07 & 0.98 & -0.70 & 0.83 & -4.20 & 0.57 \\
\hline 14 & 48 & 0.88 & 0.07 & 0.97 & -0.90 & 0.80 & -5.00 & 0.58 \\
\hline 4 & 9 & -2.61 & 0.12 & 0.93 & -0.80 & 0.45 & -3.20 & 0.30 \\
\hline 3 & 7 & 0.03 & 0.07 & 0.91 & -3.60 & 0.77 & -4.80 & 0.56 \\
\hline 1 & 3 & 0.21 & 0.07 & 0.87 & -5.00 & 0.72 & -6.40 & 0.60 \\
\hline 18 & 74 & 0.14 & 0.07 & 0.74 & -9.90 & 0.63 & -8.60 & 0.67 \\
\hline 11 & 41 & -1.17 & 0.07 & 0.69 & -9.50 & 0.45 & -7.00 & 0.58 \\
\hline 9 & 35 & 1.35 & 0.07 & 0.60 & -9.90 & 0.48 & -9.90 & 0.79 \\
\hline 20 & 77 & 1.35 & 0.07 & 0.53 & -9.90 & 0.41 & -9.90 & 0.83 \\
\hline $\mathbf{M}$ & & 0.00 & 0.09 & 1.01 & -0.40 & 1.01 & -1.10 & \\
\hline D. E. & & 1.81 & 0.05 & 0.24 & 5.90 & 0.59 & 5.90 & \\
\hline
\end{tabular}

$N=1260$ 
valores inferiores a los criterios de exclusión, por lo que los restantes 18 ítems no cumplen con el modelo de Rasch.

La calibración de los ítems del Estilo de Aprendizaje Reflexivo, presentado en la Tabla 4, indica que los parámetros del ítem (b) o dificultad, alcanzan valores que fluctúan entre los niveles muy fáciles (-2.30), como es el caso del ítem 69, y los niveles muy difíciles (4.07), como es el caso del ítem 79, lo cual implica la presencia de un espectro relativamente grande de los niveles evaluados en el constructo. También se encuentra que en la mayoría de ítems el error de estimación (E.E.) es pequeño. El análisis de la revisión de los errores Infit y Outfit, permite revelar que solamente los ítems 16, 44, 49 y 79 presen$\tan$ valores inferiores que los criterios de exclusión, de manera que los restantes 16 ítems no cumplen con el modelo de Rasch.

Tabla 4

Análisis de la calibración de los ítems del Estilo de Aprendizaje Reflexivo del CHAEA

\begin{tabular}{rrrrrrrrr}
\hline \multirow{2}{*}{ Núm. } & \multirow{2}{*}{ Ítem } & \multirow{2}{*}{$\mathbf{b}$} & E. E. & \multicolumn{2}{c}{ Infit } & \multicolumn{2}{c}{ Outfit } & rpbis \\
\cline { 5 - 8 } & & & & MNSQ & ZSTD & MNSQ & ZSTD & \\
\hline $\mathbf{7}$ & 32 & 3.60 & 0.10 & 1.16 & 2.20 & 2.05 & 5.80 & 0.05 \\
$\mathbf{9}$ & 36 & -1.11 & 0.09 & 1.07 & 1.30 & 1.77 & 6.80 & 0.25 \\
$\mathbf{1 7}$ & 65 & 0.96 & 0.06 & 1.26 & 9.70 & 1.58 & 9.90 & 0.20 \\
$\mathbf{1 1}$ & 42 & -0.49 & 0.08 & 1.25 & 5.50 & 1.31 & 4.30 & 0.25 \\
$\mathbf{5}$ & 28 & -1.31 & 0.09 & 1.12 & 2.10 & 1.28 & 2.50 & 0.27 \\
$\mathbf{8}$ & 34 & 0.34 & 0.07 & 1.16 & 4.90 & 1.21 & 4.80 & 0.34 \\
$\mathbf{1 6}$ & 63 & -0.67 & 0.08 & 1.18 & 3.90 & 1.05 & 0.70 & 0.33 \\
$\mathbf{1}$ & 10 & -0.67 & 0.08 & 1.14 & 3.10 & 0.88 & -1.70 & 0.38 \\
$\mathbf{1 2}$ & 44 & -1.26 & 0.09 & 0.97 & -0.60 & 1.13 & 1.30 & 0.37 \\
$\mathbf{1 0}$ & 39 & 1.16 & 0.06 & 1.13 & 5.20 & 1.07 & 1.80 & 0.35 \\
$\mathbf{2 0}$ & 79 & 4.07 & 0.12 & 1.08 & 0.90 & 0.98 & 0.00 & 0.10 \\
$\mathbf{1 3}$ & 49 & -0.59 & 0.08 & 1.07 & 1.50 & 0.95 & -0.70 & 0.41 \\
$\mathbf{2}$ & 16 & 1.03 & 0.06 & 0.98 & -0.90 & 0.94 & -1.80 & 0.47 \\
$\mathbf{1 4}$ & 55 & -0.50 & 0.08 & 0.56 & -3.50 & 0.59 & -7.40 & 0.61 \\
$\mathbf{1 8}$ & 69 & -2.20 & 0.12 & 0.85 & -1.80 & 0.29 & -5.90 & 0.48 \\
$\mathbf{1 9}$ & 70 & -2.18 & 0.11 & 0.84 & -1.79 & 0.26 & -5.89 & 0.47 \\
$\mathbf{3}$ & 18 & -1.13 & 0.09 & 0.80 & -4.10 & 0.47 & -7.00 & 0.60 \\
$\mathbf{1 5}$ & 58 & 0.32 & 0.07 & 0.74 & -9.20 & 0.64 & -9.90 & 0.69 \\
$\mathbf{4}$ & 19 & 0.31 & 0.07 & 0.68 & -9.90 & 0.59 & -9.90 & 0.73 \\
$\mathbf{6}$ & 31 & 0.32 & 0.07 & 0.62 & -9.90 & 0.52 & -9.90 & 0.77 \\
\hline $\mathbf{M}$ & & 0.00 & 0.08 & 1.00 & -0.10 & 0.98 & -1.10 & \\
$\mathbf{D .} \mathbf{E}$. & & 1.59 & 0.02 & 0.19 & 5.20 & 0.46 & 5.80 & \\
\hline & & & & & & & &
\end{tabular}


Tabla 5

Análisis de la calibración de los ítems del Estilo de Aprendizaje Teórico del CHAEA

\begin{tabular}{|c|c|c|c|c|c|c|c|c|}
\hline \multirow{2}{*}{ Núm. } & \multirow{2}{*}{ Ítem } & \multirow{2}{*}{ b } & \multirow{2}{*}{ E. E. } & \multicolumn{2}{|c|}{ Infit } & \multicolumn{2}{|c|}{ Outfit } & \multirow{2}{*}{ rpbis } \\
\hline & & & & MNSQ & ZSTD & MNSQ & ZSTD & \\
\hline 7 & 21 & 6.41 & 0.29 & 1.15 & 0.70 & 3.82 & 5.90 & 0.04 \\
\hline 18 & 71 & -0.88 & 0.07 & 1.00 & 0.10 & 3.33 & 9.90 & 0.31 \\
\hline 1 & 2 & 6.33 & 0.28 & 1.16 & 0.70 & 3.18 & 4.90 & 0.05 \\
\hline 19 & 78 & 6.59 & 0.31 & 1.13 & 0.50 & 2.70 & 4.30 & 0.05 \\
\hline 17 & 66 & -1.07 & 0.07 & 1.28 & 9.40 & 1.03 & 0.30 & 0.32 \\
\hline 9 & 25 & -0.49 & 0.07 & 1.25 & 8.60 & 1.20 & 2.10 & 0.37 \\
\hline 10 & 29 & -1.84 & 0.08 & 1.12 & 3.20 & 1.07 & 0.50 & 0.28 \\
\hline 15 & 60 & 0.84 & 0.07 & 1.08 & 2.00 & 0.94 & -.90 & 0.56 \\
\hline 14 & 54 & -3.27 & 0.12 & 1.07 & 0.80 & 0.71 & -1.70 & 0.18 \\
\hline 5 & 15 & 0.24 & 0.07 & 1.03 & 1.00 & 0.98 & -0.20 & 0.53 \\
\hline 13 & 50 & -0.97 & 0.07 & 1.03 & 1.00 & 0.88 & -1.10 & 0.44 \\
\hline 11 & 33 & -1.86 & 0.08 & 1.00 & 0.10 & 0.64 & -2.80 & 0.38 \\
\hline 3 & 6 & -3.14 & 0.11 & 0.99 & -0.10 & 0.52 & -3.10 & 0.27 \\
\hline 16 & 64 & -3.14 & 0.11 & 0.99 & -0.10 & 0.52 & -3.10 & 0.26 \\
\hline 2 & 4 & -1.35 & 0.07 & 0.93 & -2.40 & 0.87 & -0.90 & 0.43 \\
\hline 20 & 80 & 1.32 & 0.08 & 0.90 & -2.20 & 0.66 & -4.80 & 0.67 \\
\hline 4 & 11 & -1.41 & 0.07 & 0.85 & -5.40 & 0.64 & -2.80 & 0.49 \\
\hline 12 & 45 & -1.75 & 0.07 & 0.78 & -6.80 & 0.54 & -3.80 & 0.78 \\
\hline 6 & 17 & -3.16 & 0.11 & 0.73 & -3.60 & 0.28 & -5.50 & 0.37 \\
\hline 8 & 23 & 2.68 & 0.10 & 0.35 & -9.90 & 0.14 & -9.30 & 0.05 \\
\hline$M$ & & 0.00 & 0.11 & 0.99 & -0.10 & 1.23 & -0.06 & \\
\hline D. E. & & 3.10 & 0.08 & 0.20 & 4.30 & 1.06 & 4.30 & \\
\hline
\end{tabular}

$N=1260$

En lo que corresponde al análisis de los ítems del Estilo de Aprendizaje Teórico (tabla 5), se aprecia que los parámetros del ítem (b) o dificultad, alcanzan valores que oscilan entre los niveles muy fáciles (-3.27), como en el ítem 54, y los niveles muy difíciles (6.59), como en el ítem 78, lo cual indica la existencia de un espectro relativamente grande de los niveles evaluados en el constructo. En la mayoría de ítems el error de estimación (E. E.) es pequeño. El análisis de la revisión de los errores Infit y Outfit revela que solamente los ítems $15,50,54$ y 60 presentan valores inferiores que los criterios de exclusión, de tal forma que los restantes 16 ítems no cumplen con el modelo de Rasch. 
Los resultados de la calibración de los ítems del Estilo de Aprendizaje Pragmático presentado en la tabla 6 indican que los parámetros del ítem (b) o dificultad muestran valores que varían entre los niveles muy fáciles (-4.44), como es el caso del ítem 22, y los niveles muy difíciles (3.28), como es el caso del ítem 1, lo cual corresponde a un espectro relativamente grande de los niveles evaluados en el constructo. También se encuentra que en la mayoría de ítems el error de estimación (E. E.) es pequeño. La revisión de los errores Infit y Outfit permite revelar que solamente el ítem 14 presenta valores inferiores

Tabla 6

Análisis de la calibración de los ítems del Estilo de Aprendizaje Pragmático del CHAEA

\begin{tabular}{|c|c|c|c|c|c|c|c|c|}
\hline \multirow{2}{*}{ Núm. } & \multirow{2}{*}{ Ítem } & \multirow{2}{*}{ b } & \multirow{2}{*}{ E. E. } & \multicolumn{2}{|c|}{ Infit } & \multicolumn{2}{|c|}{ Outfit } & \multirow{2}{*}{ rpbis } \\
\hline & & & & MNSQ & ZSTD & MNSQ & ZSTD & \\
\hline 5 & 22 & -4.44 & 0.10 & 0.96 & -1.00 & 3.56 & 5.60 & 0.11 \\
\hline 13 & 56 & -1.86 & 0.07 & 1.02 & 0.03 & 2.95 & 7.80 & 0.12 \\
\hline 1 & 1 & 3.28 & 0.09 & 2.01 & 9.90 & 1.04 & 0.30 & 0.25 \\
\hline 15 & 59 & 0.48 & 0.07 & 1.74 & 9.90 & 1.77 & 9.70 & 0.15 \\
\hline 18 & 72 & 2.53 & 0.07 & 0.93 & -1.20 & 1.51 & 4.40 & 0.55 \\
\hline 2 & 8 & -0.62 & 0.11 & 1.24 & 8.40 & 1.46 & 3.70 & 0.26 \\
\hline 8 & 38 & 0.90 & 0.07 & 1.16 & 4.40 & 0.97 & -0.40 & 0.50 \\
\hline 14 & 57 & -2.50 & 0.09 & 1.15 & 1.80 & 1.04 & 0.30 & 0.12 \\
\hline 11 & 57 & -1.18 & 0.07 & 1.10 & 2.70 & 0.76 & -1.70 & 0.33 \\
\hline 17 & 68 & 2.53 & 0.07 & 1.09 & 1.60 & 0.59 & -4.80 & 0.61 \\
\hline 16 & 62 & 0.20 & 0.07 & 1.07 & 2.40 & 1.06 & 0.08 & 0.46 \\
\hline 3 & 12 & 0.56 & 0.07 & 0.96 & -1.40 & 0.85 & -2.50 & 0.56 \\
\hline 7 & 30 & -0.29 & 0.07 & 0.88 & -5.20 & 0.66 & -4.10 & 0.54 \\
\hline 4 & 14 & 0.52 & 0.07 & 0.84 & -5.60 & 0.72 & -4.80 & 0.62 \\
\hline 6 & 24 & -1.20 & 0.08 & 0.77 & -7.20 & 0.51 & -4.00 & 0.49 \\
\hline 12 & 53 & -2.67 & 0.12 & 0.76 & -2.90 & 0.28 & 5.50 & 0.36 \\
\hline 9 & 40 & -1.71 & 0.08 & 0.74 & -5.80 & 0.43 & -4.40 & 0.45 \\
\hline 10 & 47 & 0.16 & 0.07 & 0.70 & -9.90 & 0.57 & -7.00 & 0.66 \\
\hline 19 & 73 & 2.65 & 0.09 & 0.50 & -9.90 & 0.27 & -9.90 & 0.81 \\
\hline 20 & 76 & 2.65 & 0.09 & 0.50 & -9.90 & 0.27 & -9.90 & 0.81 \\
\hline$M$ & & 0.00 & 0.09 & 1.00 & -0.90 & 1.06 & -1.30 & \\
\hline D. E. & & 2.03 & 0.04 & 0.35 & 6.10 & 0.84 & 5.30 & \\
\hline
\end{tabular}

$N=1260$ 
que los criterios de exclusión, por lo que los 19 ítems restantes no cumplen con el modelo de Rasch.

Evaluación de la unidimensionalidad a través del análisis de los componentes principales de los residuos

En el análisis de la unidimensionalidad de los 20 ítems del Estilo de Aprendizaje Activo (tabla 7), se encuentra que el factor 1 , después de calcular el modelo de
Rasch, explicó el 4.3 de las 20 unidades de varianza residual, y la proporción obtenida de las unidades de varianza explicada por persona en el criterio fue de 63.0/1, por lo que se procedió a continuar el análisis debido a la posibilidad de identificar la existencia de más de un factor.

Los ítems seleccionados fueron aquellos que alcanzaron un valor absoluto mayor o igual a 0,19 . Los ítems con cargas positivas seleccionados fueron el $77,13,75,35,74,51,20$ y 26 , y los ítems

Tabla 7

Estadísticas del Factor 1 extraído por el análisis de los componentes principales de los residuos de los 20 ítems del Estilo de Aprendizaje Activo del CHAEA

\begin{tabular}{rcccc}
\hline Ítem & $\begin{array}{c}\text { Saturación } \\
\text { factorial }\end{array}$ & Medida & $\begin{array}{c}\text { Infit } \\
\text { MNSQ }\end{array}$ & $\begin{array}{c}\text { Outfit } \\
\text { MNSQ }\end{array}$ \\
\hline 26 & 0.85 & -2.48 & 1.12 & 0.92 \\
20 & 0.66 & -1.73 & 1.32 & 1.67 \\
51 & 0.53 & -1.70 & 1.31 & 1.40 \\
74 & 0.50 & 0.14 & 0.74 & 0.63 \\
35 & 0.39 & 1.35 & 0.60 & 0.48 \\
75 & 0.36 & 1.87 & 1.05 & 1.38 \\
13 & 0.30 & 1.00 & 1.30 & 1.35 \\
77 & 0.23 & 1.35 & 0.53 & 0.41 \\
5 & 0.14 & 0.99 & 1.05 & 1.30 \\
9 & 0.04 & -2.61 & 0.93 & 0.45 \\
\hline 61 & -0.68 & 1.37 & 1.13 & 1.01 \\
46 & -0.67 & 0.91 & 0.98 & 0.83 \\
48 & -0.61 & 0.88 & 0.97 & 0.80 \\
41 & -0.56 & -1.17 & 0.69 & 0.45 \\
3 & -0.54 & 0.21 & 0.87 & 0.72 \\
67 & -0.40 & 1.47 & 1.45 & 1.32 \\
7 & -0.29 & 0.03 & 0.91 & 0.77 \\
37 & -0.05 & 0.15 & 1.03 & 0.91 \\
27 & -0.05 & -4.79 & 0.98 & 0.37 \\
43 & -0.02 & 2.76 & 1.23 & 2.97 \\
\hline
\end{tabular}

$N=1260$ 
seleccionados con cargas negativas fueron el 7, 67, 3, 41, 48, 46, y 61 .

Los dos conjuntos de ítems fueron sometidos de nuevo al modelo de Rasch de forma independiente, para realizar un nuevo cálculo de las puntuaciones de los sujetos y de las puntuaciones totales. Las puntuaciones de los ítems positivos presentan una media de 1.50 $(\mathrm{DE}=1.20)$ y las puntuaciones de los ítems negativos alcanzan una media de $-0.48(\mathrm{DE}=2.15)$.
La correlación de Pearson entre ambos tipos de puntajes fue de $\mathrm{r}=0.30$, por lo que el resultado fue corregido con la fórmula profética de SpearmanBrown, por lo que se alcanza un valor de $r=0.46$. Como el coeficiente fue inferior al criterio de 0.51 (Wright, 2000), se puede concluir que el Estilo de Aprendizaje Activo del CHAEA no es unidimensional.

En lo que concierne al análisis de los ítems del Estilo de Aprendizaje Activo,

Tabla 8

Estadísticas del Factor 1 extraído por el análisis de los componentes principales de los residuos de los 20 ítems del Estilo de Aprendizaje Pragmático del CHAEA

\begin{tabular}{rcccc}
\hline Ítem & $\begin{array}{c}\text { Saturación } \\
\text { factorial }\end{array}$ & Medida & $\begin{array}{c}\text { Outfit } \\
\text { MNSQ }\end{array}$ & $\begin{array}{c}\text { Outfit } \\
\text { MNSQ }\end{array}$ \\
\hline 6 & 0.89 & -1.86 & 1.02 & 2.95 \\
38 & 0.71 & 0.90 & 1.16 & 0.97 \\
8 & 0.67 & -0.62 & 1.24 & 1.46 \\
72 & 0.33 & 2.53 & 0.93 & 1.51 \\
59 & 0.29 & 0.48 & 1.74 & 1.77 \\
24 & 0.20 & -1.20 & 0.77 & 0.51 \\
40 & 0.19 & -1.71 & 0.74 & 0.43 \\
53 & 0.10 & -2.67 & 0.76 & 0.28 \\
22 & 0.03 & -4.44 & 0.96 & 3.56 \\
\hline 73 & -0.74 & 2.65 & 0.50 & 0.27 \\
76 & -0.74 & 2.65 & 0.50 & 0.27 \\
68 & -0.65 & 2.53 & 1.09 & 0.59 \\
1 & -0.61 & 3.28 & 2.01 & 1.04 \\
30 & -0.38 & -0.29 & 0.88 & 0.66 \\
52 & -0.30 & -1.18 & 1.10 & 0.76 \\
57 & -0.22 & -2.50 & 1.15 & 1.04 \\
62 & -0.16 & 0.20 & 1.07 & 1.06 \\
47 & -0.12 & 0.16 & 0.70 & 0.57 \\
12 & -0.08 & 0.56 & 0.96 & 0.85 \\
14 & -0.08 & 0.52 & 0.84 & 0.72 \\
\hline
\end{tabular}

$N=1260$ 
en la tabla 8 se encuentra que el factor explicó 4.3 de las 20 unidades de varianza residual, y la proporción obtenida de las unidades de varianza explicada por persona en el criterio fue de 62.9/1; por ello, se procedió a continuar el análisis debido a la posibilidad de identificar la existencia de más de un factor.

Los ítems seleccionados con cargas positivas fueron el 40, 24, 59, 72, 8, 38 y 6. Mientras que los ítems con cargas negativas fueron el 57, 52, 30, 1, 68, 76 y 73. Al igual que en el caso anterior, los dos conjuntos de ítems fueron sometidos, de forma independiente, al modelo de Rasch. Los puntajes de los ítems positivos presentan una media de 3.26 $(\mathrm{DE}=2.88)$ y los puntajes de los ítems negativos alcanzan una media de -1.15 $(\mathrm{DE}=1.40)$.

La correlación entre ambos tipos de puntajes fue de $r=0.29$. El coeficiente, al ser corregido con la fórmula profética de Spearman-Brown, alcanza un valor de $r=0.45$, que, al ser inferior al criterio de 0.51 , permite concluir que el Estilo de Aprendizaje Pragmático del CHAEA no es unidimensional.

El análisis de los ítems del Estilo de Aprendizaje Reflexivo presentado en la tabla 9 permite apreciar que el fac-

Tabla 9

Estadísticas del Factor 1 extraído por el análisis de los componentes principales de los residuos de los 20 ítems del Estilo de Aprendizaje Reflexivo del CHAEA

\begin{tabular}{ccccc}
\hline Ítem & Saturación factorial & Medida & $\begin{array}{c}\text { Infit } \\
\text { MNSQ }\end{array}$ & $\begin{array}{c}\text { Outfit } \\
\text { MNSQ }\end{array}$ \\
\hline 63 & 0.77 & -0.67 & 1.18 & 1.05 \\
69 & 0.71 & -2.20 & 0.85 & 0.29 \\
70 & 0.71 & -2.20 & 0.85 & 0.29 \\
10 & 0.66 & -0.67 & 1.14 & 0.88 \\
65 & 0.63 & 0.96 & 1.26 & 1.58 \\
19 & 0.38 & 0.31 & 0.68 & 0.59 \\
28 & 0.33 & -1.31 & 1.12 & 1.28 \\
39 & 0.08 & 1.16 & 1.13 & 1.07 \\
\hline 55 & -0.77 & -0.50 & 0.86 & 0.59 \\
49 & -0.64 & -0.59 & 1.07 & 0.95 \\
48 & -0.60 & -1.13 & 0.80 & 0.47 \\
44 & -0.45 & -1.26 & 0.97 & 1.13 \\
32 & -0.36 & 3.60 & 1.16 & 2.05 \\
79 & -0.29 & 4.07 & 1.08 & 0.98 \\
31 & -0.27 & 0.32 & 0.62 & 0.52 \\
36 & -0.25 & -1.11 & 1.07 & 1.77 \\
34 & -0.21 & 0.34 & 1.16 & 1.21 \\
42 & -0.17 & -0.49 & 1.25 & 1.31 \\
16 & -0.13 & 1.03 & 0.98 & 0.94 \\
58 & -0.05 & 0.32 & 0.74 & 0.64 \\
\hline
\end{tabular}

$N=1260$ 
tor explicó 4.7 de 20 unidades de varianza residual, y la proporción obtenida de las unidades de varianza explicada por persona en el criterio fue de 13.0/1; por lo tanto, se procedió a continuar el análisis debido a la posibilidad de identificar la existencia de más de un factor.

Los ítems seleccionados con cargas positivas fueron el 28, 19, 65, 10, 70, 69 y 63 , mientras que los ítems con cargas negativas fueron el $34,36,31,79,32$, 44, 48, 49 y 55. Luego se procedió a calcular el Modelo de Rasch de forma separada para cada conjunto de ítems. Los puntajes de los ítems positivos pre- senta una media de $3.19(\mathrm{DE}=2.45)$ y los puntajes de los ítems negativos tuvieron una media de $-0.36(\mathrm{DE}=1.42)$.

El análisis de la correlación de Pearson entre ambos tipos de puntajes fue de $r=0.28$, que al ser corregido con la fórmula profética de SpearmanBrown, alcanza un valor de $\mathrm{r}=0.44$, que es inferior al criterio de 0.51 ; por ello, se puede concluir que el Estilo de Aprendizaje Reflexivo del CHAEA no es unidimensional.

En lo que respecta al análisis Estilo de Aprendizaje Teórico, en la tabla 10 se encuentra que el factor explicó el 3.8

Tabla 10

Estadísticas del Factor 1 extraído por el análisis de los componentes principales de los residuos de los 20 ítems del Estilo de Aprendizaje Teórico del CHAEA

\begin{tabular}{ccccc}
\hline $\begin{array}{c}\text { Saturación } \\
\text { factorial }\end{array}$ & Medida & $\begin{array}{c}\text { Infit } \\
\text { MNSQ }\end{array}$ & $\begin{array}{c}\text { Outfit } \\
\text { MNSQ }\end{array}$ & Ítem \\
\hline 0.76 & -3.14 & 0.99 & 0.52 & 6 \\
0.76 & -3.14 & 0.99 & 0.52 & 64 \\
0.65 & -1.75 & 0.78 & 0.54 & 65 \\
0.58 & 0.84 & 1.08 & 0.94 & 60 \\
0.48 & 0.24 & 1.03 & 0.98 & 15 \\
0.29 & -0.49 & 1.25 & 1.20 & 25 \\
0.11 & 1.32 & 0.90 & 0.66 & 80 \\
0.05 & 2.68 & 0.35 & 0.14 & 23 \\
0.01 & -3.16 & 0.73 & 0.28 & 17 \\
-0.67 & -1.86 & 1.00 & 0.64 & 33 \\
-0.63 & -3.37 & 1.07 & 0.71 & 54 \\
-0.56 & -1.41 & 0.85 & 0.64 & 11 \\
-0.41 & -0.97 & 1.03 & 0.88 & 50 \\
-0.38 & -1.07 & 1.28 & 1.03 & 66 \\
-0.31 & -1.35 & 0.93 & 0.87 & 4 \\
-0.06 & -0.88 & 1.00 & 3.33 & 71 \\
-0.04 & -1.84 & 1.12 & 1.07 & 29 \\
-0.04 & 6.59 & 1.13 & 2.70 & 78 \\
-0.03 & 6.33 & 1.16 & 3.18 & 21 \\
-0.02 & 6.41 & 1.15 & 3.82 & \\
\hline
\end{tabular}


de 20 unidades de varianza residual, y la proporción obtenida de las unidades de varianza explicada por persona en el criterio fue de 63.0/1. Se procedió a continuar el análisis debido a la posibilidad de identificar la existencia de más de un factor.

Los ítems seleccionados con cargas positivas fueron el 25, 15, 60, 65, $64 \mathrm{y}$ 6 , mientras que los ítems con cargas negativas fueron el 4, 66, 50, 11, 54 y 33. Después se procedió a calcular el modelo de Rasch para cada conjunto de ítems de forma separada. Los puntajes de los ítems positivos presentaron una media de 2.64 ( $\mathrm{DE}=1.97)$ y los puntajes de los ítems negativos tuvieron una media de -1.38 ( $\mathrm{DE}=2.23)$.

El análisis de la correlación de Pearson entre ambos tipos de puntaje fue de $r=0.40$, que al ser corregido con la fórmula profética de Spearman-Brown, alcanza un valor de $\mathrm{r}=0.33$. Como el coeficiente fue inferior al criterio de 0.51, se puede concluir que el Estilo de Aprendizaje Teórico del CHAEA no es unidimensional.

\section{DISCUSIÓN}

Los resultados de los estilos de aprendizaje de Honey y Alonso efectuados bajo el contexto de la Teoría Clásica de los Tests realizados a nivel de análisis de ítems, así como la confiabilidad por consistencia interna de cada una de las escalas, ha permitido observar que las correlaciones ítem-test corregidas su- peraron el criterio propuesto por Kline (1986) de ser mayores de 0.20, en tanto que los coeficientes de confiabilidad de Kuder Richardson 20 obtuvieron valores que pueden clasificarse como adecuados. Estos hallazgos concuerdan con los reportes de Alonso, Gallego y Honey $(2002,1994)$ y Escurra (2005).

El análisis de la validez realizada en su modalidad de evidencia de validez de constructo, reporta que el análisis factorial confirmatorio permite corroborar que el modelo de un factor se encuentra de forma consistente y adecuada en los datos obtenidos en la investigación, lo cual coincide con los hallazgos alcanzados en los estudios realizados por Escurra (2005) y por Alonso, Gallego y Honey $(2002,1994)$.

En términos generales se puede indicar que el CHAEA presenta evidencias de validez de constructo y confiabilidad por consistencia interna, con lo cual se cumplen los requerimientos psicométricos básicos de la Teoría Clásica de los Tests para este tipo de instrumentos, tal como lo proponen Delgado, Escurra y Torres (2006); Anastasi y Urbina (1998); Martínez (1996); Muñiz (1994, 1996, 1996a, 1997); Aiken (1996) y Brown (1993), entre otros.

Respecto al análisis de los Estilos de Aprendizaje del CHAEA a través de la Teoría de la Respuesta al Ítem con el Modelo de Rasch, por ser un modelo paramétrico, requiere el cumplimiento de requisitos más exigentes (De Ayala, 2009). 
Los resultados con relación a los análisis gráficos permiten identificar que los ítems de los estilos cubren de manera adecuada el espectro del constructo que se evalúa. Este es un resultado que refleja la posibilidad de obtener valoraciones que cumplan un rango amplio de las actividades que definen los constructos analizados, tal como lo proponen De Ayala (2009); Furr y Bacharach (2008); Embretson y Reise (2000), además de Van Der Linden y Hambleton (1997).

En relación con los análisis de las funciones de información los hallazgos son positivos, pues también el rango de los valores con puntajes precisos presenta una amplitud adecuada, tal como lo indican De Ayala (2009), Muñiz $(1997,1996)$ y Hambleton, Swaminathan y Rogers (1991), pero el rango de los puntajes con mayores errores e imprecisiones se presenta en los valores de los extremos inferiores y superiores.

Los resultados relacionados con la calibración de cada uno de los estilos de aprendizaje demuestran que los errores Infit y Outfit predominan en todos los casos, lo cual indicaría que los sujetos utilizan diferentes patrones de respuesta a los ítems que conforman los instrumentos; esto revelaría la existencia de la medición de varios constructos de manera simultánea, o también la existencia de condicionalidad en los ítems evaluados, tal como sugieren Bond y Fox (2001), Embretson y Reise (2000) y Van Der Linden y Hambleton (1997).
Los resultados relacionados con la unidimensionalidad de los constructos efectuados a través del análisis de los componentes principales de los residuos (Linacre, 2007; Bond y Fox, 2001; Wright, 2000; Smith y Miao, 1994) permitieron corroborar que en cada uno de los estilos existen, por lo menos, dos variables latentes, que son medidas y que por lo tanto el instrumento no se comporta de acuerdo con el modelo de Rasch por ser multidimensional.

Por otro lado, el análisis comparativo de los modelos permite revelar que la Teoría Clásica de los Tests, por ser un modelo poco exigente en términos psicométricos, es más fácil de ser cumplido en los diferentes instrumentos psicológicos, mientras que la exigencia de la Teoría de la Respuesta al Ítem a través del Modelo de Rasch requiere el cumplimiento estricto de sus supuestos.

Esto es más difícil de obtenerse por los instrumentos psicológicos, pero aquellos que pueden cumplirlos permiten obtener mediciones de una mayor calidad, tal como reportan los estudios desarrollados por Adedoyin, Nenty y Chilisa (2008); Prieto y Delgado (2003); Fan (1998) y Gutiérrez y Gonzales (1992) en lo que corresponde a su aplicación a pruebas de rendimiento; así como en los estudios realizados por Prieto, Alonso y Lamarca (2003), y Jackson, Draugalis, Snack, Zachny y D'Agostino (2002) en instrumentos de rasgos psicológicos (De Ayala, 2009). 
De los hallazgos reportados se deriva la imperiosa necesidad de realizar estudios que permitan adaptar las pruebas psicológicas que se usan de forma profesional por los psicólogos de nuestro medio, haciendo uso de metodologías psicométricas sustentadas en la Teoría de la Respuesta al Ítem, como la del modelo de Rasch, de manera que se puedan obtener mediciones más precisas y de calidad.

\section{CONCLUSIONES}

1. El Estilo de Aprendizaje Activo del CHAEA presenta confiabilidad por consistencia interna.

2. El Estilo de Aprendizaje Reflexivo del CHAEA presenta confiabilidad por consistencia interna.

3. El Estilo de Aprendizaje Teórico del CHAEA presenta confiabilidad por consistencia interna.

4. El Estilo de Aprendizaje Pragmático del CHAEA presenta confiabilidad por consistencia interna.

5. El CHAEA presenta evidencias de validez de constructo bajo el modelo de la Teoría Clásica de los Tests.

6. Los ítems del Estilo de Aprendizaje Activo del CHAEA no cumplen con los supuestos teóricos del modelo de Rasch.

7. Los ítems del Estilo de Aprendizaje Reflexivo del CHAEA no cumplen con los supuestos teóricos del modelo de Rasch.
8. Los ítems del Estilo de Aprendizaje Teórico del CHAEA no cumplen con los supuestos teóricos del modelo de Rasch.

9. Los ítems del Estilo de Aprendizaje Pragmático del CHAEA no cumplen con los supuestos teóricos del modelo de Rasch.

10.El CHAEA cumple con los requerimientos de la TCT, pero no cumple con los supuestos teóricos del modelo de Rasch.

11. El CHAEA permite obtener solo mediciones ordinales con un error de medición mayor.

\section{RECOMENDACIONES}

A partir de los resultados, la discusión y las conclusiones se proponen las siguientes recomendaciones:

1. Desarrollar nuevas investigaciones con el CHAEA pero aplicando modelos de la Teoría de la Respuesta al Ítem que permitan manejar la multidimensionalidad de los ítems.

2. Realizar investigaciones similares pero considerando otras muestras, con el fin de establecer la generalidad de los resultados encontrados.

3. Aplicar los modelos de la Teoría de la Respuesta al Ítem como procedimiento metodológico, con el fin de evaluar los instrumentos psicológicos utilizados en nuestro medio, tanto para pruebas de ejecución máxima o rendimiento como para pruebas de ejecución típica o que midan rasgos psicológicos. 


\section{REFERENCIAS}

Adán, M. I. (2007). Los estilos de aprendizaje en la orientación y tutoría en Bachillerato. Revista Complutense de Educación. Vol. 19 (1), 59-76.

Adedoyin, O. O., Nenty, H. J., \& Chilisa, B. (2008). Investigating the invariance of item difficulty parameter estimates based on CTT and IRT. Educational Research and Review, 3 (2), 83-93. Obtenido el 10 de agosto del 2010, de <http://www.academicjournals.org/ERR/PDF/Pdf\%20 2008/Mar/Adedoyin\%20et\%20al. pdf $>$.

Aiken, L. R. (1996). Tests psicológicos y evaluación. México D. F.: Prentice Hall International.

Alarcón, R. (2008). Métodos y diseños de investigación del comportamiento. Lima: Universidad Ricardo Palma.

Allen, M. J. \& Yen, W. M. (2002). Introduction to measurement theory. Illinois: Waveland Press.

Alonso, C., Gallego D. J., \& Honey, P. (1994). Cuestionario de HoneyAlonso de Estilos de Aprendizaje. Madrid: Instituto de Ciencias de la Educación (ICE).

Alonso, C., Gallego, D. J., \& Honey, P. (2002). Los estilos de aprendizaje. Procedimientos de diagnóstico y mejora. Bilbao: Mensajero.
American Psychological Association - APA (1986). Standards for educational and psychological testing. Washington: APA.

Anastasi, A. \& Urbina, S. (1998). Tests psicológicos. México D. F.: Prentice Hall.

Andrich, D. (1988). Rasch models for measurement. Londres: Sage Publications Ltd.

Arbuckle, J. L. (2007). Amos 16.0. User's guide. Spring House, PA: Amos Development Corporation.

Beltrán, J. (1996). Estrategias de aprendizaje. En Beltrán, J. y Genovard, C. (Eds.). Psicología de la instrucción. Variable y procesos básicos. Madrid: Síntesis.

Blunch, N. J. (2008). Introduction to structural equation modeling, using SPSS and AMOS. Los Ángeles: Sage.

Bond, T. G. \& Fox, C. M. (2001). Applying the Rasch model, fundamental measurement in the human sciences. Nueva Jersey: Lawrence Erlbaum Editors.

Brown, F. (1993). Principios de la medición en Psicología y Educación. México D. F.: El Manual Moderno.

Brown, T. (2006). Confirmatory factor analysis for applied research. Nueva York: The Guilford Press. 
Bustinza, C., Durán, D., \& Quintasi, J. (2006). Diagnóstico de estilos de aprendizaje de estudiantes del IV ciclo de la especialidad de Educación Inicial. Lima: Tarea. Obtenido el 9 de agosto del 2010, de <http://www. tarea.org.pe/images/ClaudiaBustinza_DiagnosticoEstilos.pdf $>$.

Byrne, B. M. (2001). Structural equation modelling with AMOS: Basic concepts, applications and programming. Nueva Jersey: Lawrence Erlbaum Associates Publishers.

Camarero, F., Martín del Buey, F., \& Herrero, J. (2000). Estilos y estrategias de aprendizaje en estudiantes universitarios. Psicothema, 12 (4), 615-622.

Contreras, Y. (2007). Los estilos de aprendizaje en los alumnos de nivel superior. IX Congreso Nacional de Investigación Educativa. Obtenido el 15 de agosto del 2010, de $<$ http://www.comie.org.mx/congreso/memoria/va/ponencias/at01/ PRE1178817850.pdf $>$.

Cortada, N. (1999). Teorías psicométricas y construcción de tests. Buenos Aires: Lugar Editorial S. A.

Cortada, N. (2000). Técnicas psicológicas de evaluación y exploración. México D. F.: Trillas.

De Ayala, R. J. (2009). The Theory and Practice of Item Response Theory. Nueva York: The Guilford Press.
Delgado, A. E., Escurra, L. M., \& Torres, W. (2006). La medición en Psicología y Educación: Teoría y aplicaciones. Lima: Hozlo.

Du Toit, M. (2003). IRT from SSI. Lincolwood: Scientific Software International Inc.

Elorza, H. (2007). Estadísticas para las ciencias sociales, del comportamiento y de la salud. México D. F.: Cengage Learning.

Embretson, S., \& Reise, S. S. (2000). Item response theory for psychologists. Nueva Jersey: Lawrence Erlbaum Associates Publishers.

Escurra, L. M., \& Delgado, A. E. (1994). El modelo de un parámetro Logist de Rasch. Boletín de la Unidad de Post Grado de Psicología 5 (1), 25-30.

Escurra, L. M. (2005). Estilos, estrategias de aprendizaje y rendimiento académico en estudiantes universitarios de Lima metropolitana. Informe de Investigación. Lima: Universidad de Lima. Inédito.

Fan, X. (1998). Item Response Theory and Classical Test Theory: An empirical comparison of the item/person statistics. Educational and Psychological Measurement, 58 (3), 357374. doi:10.1177/0013164498058003 001 .

Furr, M. R., \& Bacharach, V. R. (2008). Psychometrics: An introduction. Los Ángeles: Sage Publications. 
Gómez del Valle, M. et al. (2003). Identificación de los estilos de aprendizaje predominantes en estudiantes de magisterio de la Facultad de Ciencias de la Educación de la Universidad de Cádiz. Revista Electrónica Interuniversitaria de Formación del Profesorado, 6 (2). Obtenido el 20 de agosto del 2010, de $<$ http://www. aufop.com/aufop/uploaded_files/articulos/1227721086.pdf $>$.

Gómez, J., \& Yacarini, E. E. (2006). Estilos de aprendizaje y rendimiento académico en estudiantes de la Universidad Católica Santo Toribio de Mogrovejo. Monografia. Obtenido el 15 de junio del 2010, de <http:// www.ilustrados.com/publicaciones/ EEuZkEkVAEnQMjJTmP.php>.

Gutiérrez, L., \& González-Tablas, M. M. (1992). Calibración de los ítems del PMA-E desde el modelo de Rasch. Revista de Psicología General y Aplicada, 453, 275-284. Obtenido el 18 de junio del 2010, de $<$ http://dialnet.unirioja.es/servlet/ articulo? codigo $=2378331>$.

Hambleton, R., Swaminathan, H., \& Rogers, J. (1991). Fundamentals of Item Response Theory. Londres: Sage Publications Ltd.

Hancock, G. R., \& Mueller, R. O. (2006). Structural Equation Modeling, Second course. Madison: Information Age Publishing Inc.

Hernández, S., Fernández, C., \& Baptista, L. (2006). Metodología de la investigación. México D. F.: McGraw-Hill.

Honey, O., \& Mumford, A. (1986). The manual of learning styles. Maidenhead: Peter Honey.

Jackson, T. R., Draugalis, L. R., Slack, M. K., Zachry, W. M., \& D'Agostino, J. (2002). Validation of authentic performance assesment: A process suited for Rasch Modeling. American Journal of Pharmaceutical Education, 66, 233-243.

Kline, P. (1986). A handbook of construction. Introduction to psychometric design. Londres: Methuen.

Kline, P. (1993). The handbook of psychological testing. Londres: Routledge.

Kolb, D. (1984). Experiential learning: Experience as the source of learning and development. Nueva Jersey: Prentice-Hall.

Linacre, J. M. (2007). Winsteps: Rasch measurement computer program. Chicago: Winsteps.

López Pina, J. A. (1995). Teoría de la Respuesta al Ítem: Fundamentos. Barcelona: Promociones y Publicaciones Universitarias.

Lord, F., \& Novick, M. (1974). Statistical theories of mental tests scorer. Massachusetts: Addison Wesley.

Luengo González, R., \& González Gómez, J. J. (2005). Relación entre los estilos de aprendizaje, el rendimien- 
to en matemáticas y la elección de asignaturas optativas en alumnos de E.S.O. Revista Electrónica de Investigación y Evaluación Educativa, 11, (2). Obtenido el 20 de noviembre del 2010, de <http://www.uv.es/Relieve/ v11n2/relievev11n2_4.htm>.

Magnusson, D. (1976). Teoría de los tests. México D. F.: Trillas.

Marín, G. (1986). Consideraciones metodológicas básicas para conducir investigaciones psicológicas en América Latina. Acta Psiquiátrica y Psicológica de América de Latina, 32, 183-192.

Martínez, R. (2006). Psicometría: Teoría de los tests psicológicos y educativos. Madrid: Alianza Editorial.

Montesinos, J. (2008). El análisis de reactivos con el modelo Rasch. Manual técnico A. México D. F.: Universidad de Sonora.

Muñiz, J. (1990). Teoría de respuesta a los items: Un nuevo enfoque en la evolución psicológica y educativa. Madrid: Pirámide.

Muñiz, J. (1994). Teoría Clásica de los Tests. Madrid: Pirámide.

Muñiz, J. (1996). Psicometría. Madrid: Universitas.

Muñiz, J. (1996a). Teoría Clásica de los Tests. Madrid: Pirámide.

Muñiz, J. (1997). Introducción a la Teoría de Respuesta a los Ítems. Madrid: Pirámide.
Nunnally, J., \& Bernstein, I. (1995). Teoría psicométrica. México D. F.: McGraw-Hill.

Ordóñez, F. J., Rosety-Rodríguez, M., \& Rosety-Plaza, M. (2003). Análisis de los estilos de aprendizaje predominantes entre los estudiantes de Ciencias de la Salud. Revista Electrónica Semestral de Enfermería, 3. Obtenido el 20 de mayo del 2010, de $<$ www.revistas.um.es/eglobal/article/download/619/645>.

Prieto, G., \& Delgado, A. R. (2003). Análisis de un test mediante el modelo de Rasch. Psicothema, 15 (1). 94-100. Obtenido el 20 de mayo del 2010, de <http://www.psicothema. com/pdf/1029.pdf $>$.

Prieto, L., Alonso, J., \& Lamarca R. (2003). Classical test theory versus Rasch análisis for quality of life questionnaire reduction. Health Quality of Life Outcomes, 1 (27), doi:10.1186/1477-7525-1-27.

Rasch, G. (1980). Probabilistic models for some intelligence and attainment test Chicago: Illinois: University of Chicago Press.

Raykov, T., \& Marcoulides, G. A. (2000). A first course in structural equation modeling. Nueva Jersey: Lawrence Erlbaum.

Sánchez, C. H., \& Reyes, M. C. (2006). Metodología y diseños en la investigación cientifica aplicados a la Psicología, la Educación y las Ciencias 
Sociales. Lima: Universidad Ricardo Palma.

Santisteban Requena, C. (2009). Principios de Psicometría. Madrid: Síntesis.

Sckumacker, R. E., \& Lomas, R. G. (2004). A beginner's guide to structural equation modeling. Nueva Jersey: Lawrence Erlbaum Associates Publishers.

Smith, R. M., \& Miao, C. Y. (1994). Assessing unidimensionality for Rasch measurement. Chapter 18 in Objective Measurement: Theory into Practice, Vol. 2. (316-327). M. Wilson (Ed.). Norwood, NJ: Ablex Publishing Corporation.

Thompson, B. (2008). Exploratory and confirmatory factor analysis, understanding concepts and applications. Washington D. C.: American Psychological Association.

Thorndike, R. L. (1989). Psicometría aplicada. México D. F.: Limusa.
Tristán L., A. (2001). Análisis de Rasch para todos: Una guía simplificada para evaluadores educativos. México D. F.: Ceneval.

Van Der Linden, W. J., \& Hambleton R. K. (1997). Handbook of modern item response theory. Nueva York: Springer Verlag.

Wilson, M. (2005). Constructing measures: An item response modeling approach. Londres: Lawrence Erlbaum.

Witkin, H. A., \& Goodenoug, D. R. (1991). Estilos cognitivos. Naturaleza y orígenes. Madrid: Pirámide.

Wright, B. D. (2000). Conventional factor analysis vs. Rasch residual factor analysis. Rasch Measurement Transactions, 14 (2), 753. Obtenido el 20 de julio del 2010, de <http:// www.rasch.org/rmt/rmt142m>.

Zabalza, M. A. (2002). La enseñanza universitaria, el escenario y sus protagonistas. Madrid: Narcea Ediciones. 